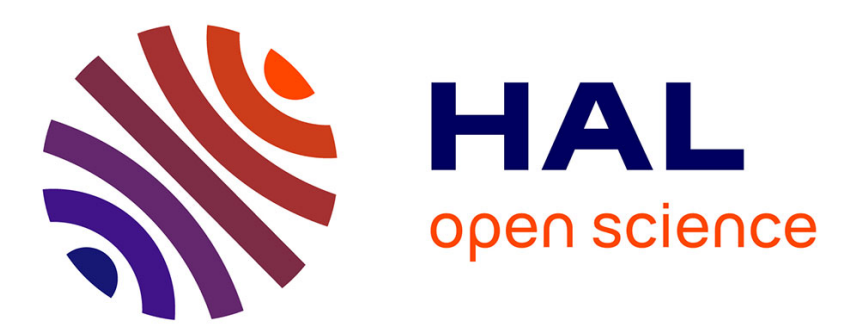

\title{
Coherent and turbulent process analysis of the effects of a longitudinal vortex on boundary layer detachment on a NACA0015 foil
}

Sébastien Prothin, Henda Djeridi, Jean-Yves Billard

\section{- To cite this version:}

Sébastien Prothin, Henda Djeridi, Jean-Yves Billard. Coherent and turbulent process analysis of the effects of a longitudinal vortex on boundary layer detachment on a NACA0015 foil. Journal of Fluids and Structures, 2014, 47, pp.2- 20. 10.1016/j.jfluidstructs.2013.08.014 hal-01059682

\section{HAL Id: hal-01059682 \\ https://hal.science/hal-01059682}

Submitted on 1 Sep 2014

HAL is a multi-disciplinary open access archive for the deposit and dissemination of scientific research documents, whether they are published or not. The documents may come from teaching and research institutions in France or abroad, or from public or private research centers.
L'archive ouverte pluridisciplinaire HAL, est destinée au dépôt et à la diffusion de documents scientifiques de niveau recherche, publiés ou non, émanant des établissements d'enseignement et de recherche français ou étrangers, des laboratoires publics ou privés. 


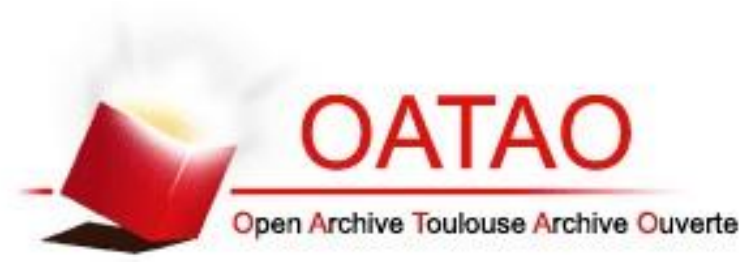

\section{Open Archive Toulouse Archive Ouverte (OATAO)}

OATAO is an open access repository that collects the work of Toulouse researchers and makes it freely available over the web where possible.

This is an author-deposited version published in: http://oatao.univ-toulouse.fr/ Eprints ID: 9867

To link to this article: DOI: 10.1016/j.jfluidstructs.2013.08.014

URL: http://dx.doi.org/10.1016/j.jfluidstructs.2013.08.014

To cite this version: Prothin, Sebastien and Djeridi, Henda and Billard, Jean-Yves Coherent and turbulent process analysis of the effects of a longitudinal vortex on boundary layer detachment on a \{NACA0015\} foil. (2014) Journal of Fluids and Structures, vol. 47. pp. 2- 20. ISSN 08899746 


\title{
Coherent and turbulent process analysis of the effects of a longitudinal vortex on boundary layer detachment on a NACA0015 foil
}

\author{
Sebastien Prothin ${ }^{a}$, Henda Djeridi ${ }^{\mathrm{b}, *}$, Jean-Yves Billard ${ }^{\mathrm{c}}$ \\ a Institut Supérieure de l'Aéronautique et de l'Espace (ISAE Toulouse), Département Aérodynamique, Energétique et Propulsion, \\ BP 54032,10 Av. Edouard Belin, 31055 Toulouse, France \\ ${ }^{\mathrm{b}}$ Laboratoire de Physique des Océans (LPO, UMR 6523, CNRS, IFREMER, IRD, UBO), Université Européenne de Bretagne, Université de Brest. \\ 6 Av. Le Gorgeu, BP 809, 29285 Brest Cedex, France \\ ' Institut de Recherche de l'Ecole Navale (IRENav EA 3634), Département M2EN, CC 400, 29240 BREST Cedex 9, France
}

A R T I C L E I N F O

Keywords:

Hub vortex

Vortex shedding

TR-SPIV

POD

\begin{abstract}
A B S T R A C T
In this paper, the influence of a single tip vortex on boundary layer detachment is studied. This study offers a preliminary approach in order to better understand the interaction between a propeller hub vortex and the rudder installed in its wake. This configuration belongs to the field of marine propulsion and encompasses such specific problem as cavitation inception, modification of propulsive performances and induced vibrations. To better understand the complex mechanisms due to propeller-rudder interactions it was decided to emphasize configurations where the hub vortex is generated by an elliptical 3-D foil and is located upstream of a 2-D NACA0015 foil at high incidences for a Reynolds number of $5 \times 10^{5}$. The physical mechanisms were studied using Time Resolved Stereoscopic Particle Image Velocimetry (TR-SPIV) techniques. Particular attention was paid to the detachment at $25^{\circ}$ incidence and a detailed cartography of the mean and turbulent properties of the wake is presented. Proper Orthogonal Decomposition (POD) analysis was applied in order to highlight the unsteady nature of the flow using phase averaging based on the first POD coefficients to characterize the turbulent and coherent process in the near wake of the rudder.
\end{abstract}

\section{Introduction}

The global aim of the present study was to analyze the flow interaction mechanisms occurring in the space between a ship's propeller and the rudder in order to improve its performance (e.g. maneuverability, stability, etc.). As noted by many authors (Tsakonas et al., 1970; Zhu and Dong, 1986; Shen et al., 1997; Kinnas et al., 2006; Felli et al., 2009) positioning the rudder behind a propeller leads to a complex series of interaction mechanisms characterized by unsteady and vortical upstream flow on the rudder, cavitation phenomena (sheet and vortex cavitation which cause structural damage, vibrations) and fatigue stresses. For large rudder angles, during lower speed maneuvering configurations, instabilities of the von Kàrmàn street type are visible in the wake of the propeller.

\footnotetext{
* Corresponding author. Present address: L.E.G.I., Grenoble-INP, UMR 5519, BP 53, 38041 Grenoble Cedex 9, France. Tel.: +33 476825063.

E-mail addresses: henda.djeridi@legi.grenoble-inp.fr, henda.djeridi@univ-brest.fr (H. Djeridi).
} 
Whilst there is a large number of studies exploring the propeller flow field, wake features and tip vortex trajectory, few analyses investigate the detached flow behind a rudder at high incidences and the influence of the upstream vortex on the unsteady separated flow. Quantifying the topology in the presence of a coherent vortex upstream leads to an improved prediction performance of the rudder interaction with the wake of the propeller.

Among the latter studies, Felli et al. (2006) measured the velocity and pressure fields behind a marine propeller using PIV techniques along with phased pressure measurements based on a reference blade position. They stressed the important contribution, made by the passage of the tip vortex to the generation of the pressure field in the propeller flow. Furthermore, via Laser Doppler Velocimetry (LDV) phase-sampling techniques and flow visualizations, Felli et al. (2009) highlighted the unsteady flow features characterizing the interaction between the rudder and the propeller. More particularly, they found a strong deformation of the velocity field and a local increase in the turbulence level when the blade tip vortex crossed the rudder. In a recent paper, Felli et al. (2011) investigated the tip vortex penetration into the rudder flow field and the rejoining process behind the trailing edge as well as the mechanism governing the spanwise displacement of the tip vortex filaments running on the pressure and suction sides of the rudder. Although this aforementioned interaction represents a better understanding of the physical mechanism in the naval propulsion domain, the study did not include any information on the topological features of the wake of the rudder at high incidences during the maneuvering process. The above review highlights the lack of experimental investigation into the unsteady flow separation on the rudder and particularly into the specific approach needed to describe the coherent motion due to the shedding vortex process.

Taking into account the complexity of the interaction mechanism of the propeller-rudder combination, the present research focuses on the presence of a single isolated hub vortex. The vortex was generated by a three dimensional NACA0020 foil located upstream of the rudder which was modeled on a two dimensional NACA0015 foil. In the case of lower speed maneuvering, an angle of attack of $25^{\circ}$ was considered, corresponding to post-stall conditions. Proper Orthogonal Decomposition (POD) was applied to the velocity field measurements and phase averaging, based on the determination of the first coefficients of POD, leads to the characterization of the vortex shedding in both cases, with and without the upstream vortex. The analysis of the flow in the near and far wake of the longitudinal vortex/NACA0015 configuration was based on:

(i) Time resolved stereoscopic PIV measurements on the upper part of the suction side of the NACA0015 foil, at various locations in the wake. To characterize the non-uniform velocity distribution due to the presence of the upstream longitudinal vortex, three PIV planes normal to the span were considered allowing us to take into account two zones referred to as "outflow" and "inflow" areas (up wash and downwash motions on the foil).

(ii) Force measurements with a balance gauge to characterize the hydrodynamic parameters of the two dimensional profile at low and high incidence.

The paper is organized as follows: Section 2 presents experimental set-up, the flow configuration and TR-SPIV measurements. Section 3 summarizes the principal results of the vortex interaction on global forces and velocity fields in the near wake of the NACA0015 foil. The results relative to the POD analysis and phase averaging are presented in Section 4. Conclusions are summarized in Section 5.

\section{Experimental set-up and measurements}

\subsection{Experimental configuration and velocity measurements}

The experiments were conducted in the hydrodynamic tunnel of the French Naval Academy (also described in Ducoin et al., 2012). This facility comprises a test section $1 \mathrm{~m}$ long with a square cross section measuring $0.192 \times 0.192 \mathrm{~m}^{2}$ in which a maximum velocity of $15 \mathrm{~ms}^{-1}$ can be achieved. The experiment is composed of two foils: the first is used as vortex generator and is a finite wing span NACA0020 foil with elliptical plane form. It will be referred to as the wing further on. Its chord, $c_{\text {ell }}$, is equal to $54 \mathrm{~mm}$ and its span $81 \mathrm{~mm}$. The second is a two dimensional NACA0015 foil (referred to as profile) of chord length $c$ equal to $100 \mathrm{~mm}$. The dimensions and relative positions of the two foils are detailed in Fig. 1(a) and (b). For this experimental configuration, $(x, y, z)$ are the curvilinear coordinates associated with the 2-D foil and $(X, Y, Z)$ are the Cartesian coordinates associated with the 3 -D foil. For the tip vortex, cylindrical coordinates $(r, \theta, z)$ are used to localize the vortex core.

The upstream flow velocity, $U_{i n f}$, is equal to $5.56 \mathrm{~ms}^{-1}$, and the resulting Reynolds number based on the profile chord length is $5.6 \times 10^{5}$. For these flow conditions the upstream turbulence intensity is $2 \%$. The blockage ratio defined as $t / H$ (where $t$ is the profile maximum thickness and $H$ is the height of the test-section) was 0.078 . The upstream hydrofoil was mounted vertically on the bottom of the test section using a pivot connection. Particular attention was paid to its anchoring to reduce any vibration as much as possible. The incidence angle is fixed with a precision of \pm 7 min. Measurements of the tangential and axial velocities in the vortex core were carried out at several positions along the axis using an LDV system for different incidence angles. This was done to control the intensity and location of the tip vortex. Its characteristics have been 
(a)
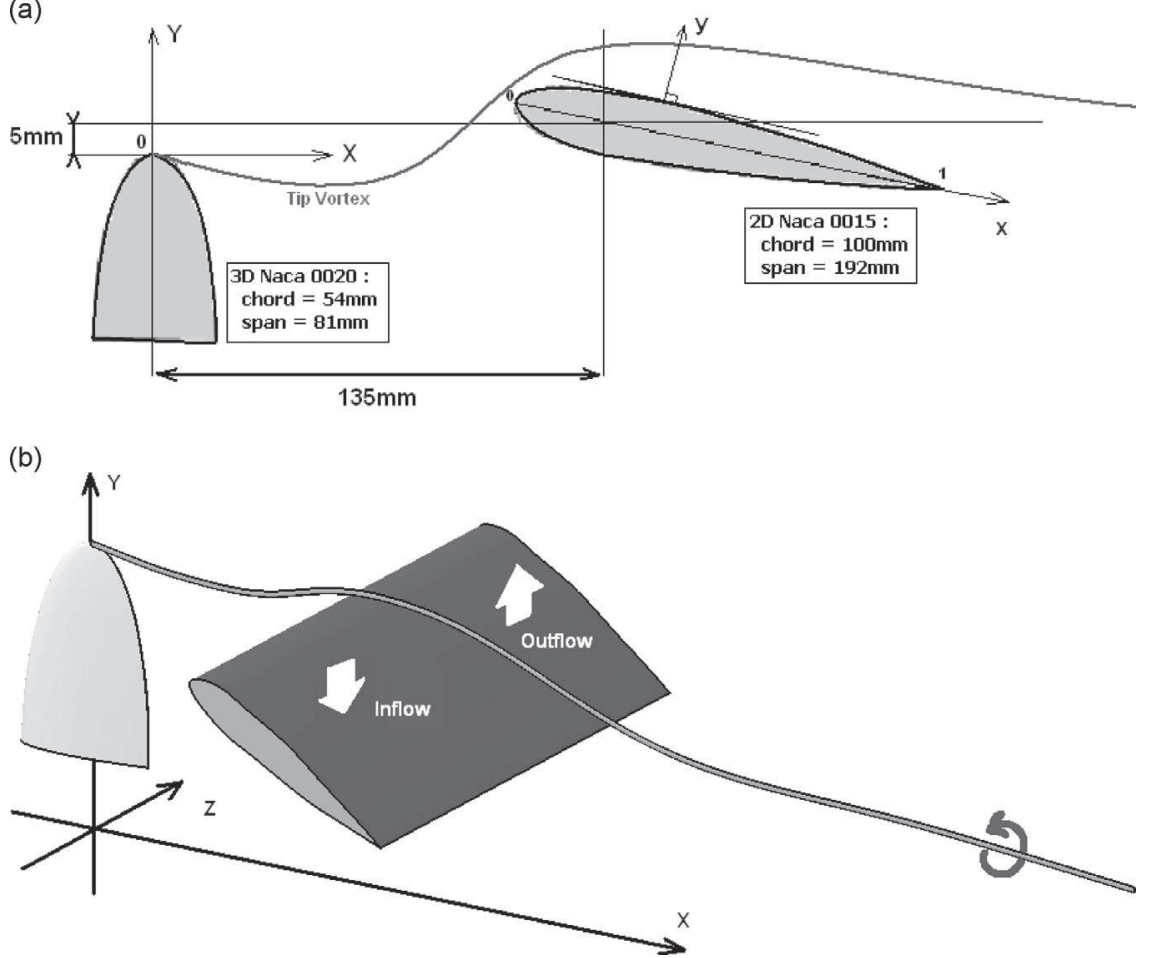

Fig. 1. (a) Dimensions of the experimental configuration and (b) relative locations of the foils.

extensively documented, and all present measurements have been compared with those obtained previously in the same test section (documented by Pichon, 1995).

We verified experimentally (using LDV) that the wake of the wing body did not disturb the flow on the suction side of the profile (see Prothin, 2011). The incidence of the wing for the generation of the tip vortex was low enough to result in a thin wake. On the upper surface of the 2-D foil the vortex generates two zones namely "outflow" area where the vortex induces a velocity directed from the profile wall and an "inflow" area with induced velocity toward the foil.

Time-resolved Stereoscopic PIV (TR-SPIV) measurements were carried out for the NACA0015 profile at $25^{\circ}$ incidence. A double pulse Nd:Yag laser (DualPower TR Lasers) was used for the experiments, delivering $2 \times 30 \mathrm{~mJ}^{2}$. Silver glass particles of diameter $10 \mu \mathrm{m}$ were used as flow seeding, with a $1 \mathrm{~mm}$ thick light sheet. Two SpeedSense 9072 cameras were used with a resolution of $1280 \times 800$ pixels, recording double full-frame particle images. The cameras were equipped with a $60 \mathrm{~mm}$ objective lens at a diaphragm aperture of 1.2. Both camera and laser were operated at a frequency of $1000 \mathrm{~Hz}$. The set-up is shown in Fig. 2(a).

The measurement area, which is a plane perpendicular to the foil at a given spanwise distance, is divided into three sections $P 1, P 2$ and $P 3$ each located further downstream of the profile (see Fig. 2(b)). There are three spanwise planes considered. One is along the centerline of the profile with another on either side in order to capture the 3-D effects of the upstream vortex (only the off center planes are schematized in Fig. 2(b)). This configuration allows the characterization of the near wake area $(P 1-P 2)$ and the far wake area $(P 3)$. To characterize the non-uniform velocity distribution due to the presence of the vortex, two PIV-planes were considered for inflow and outflow configurations at $Z / c=-0.48$ and 0.48 respectively. The overall size of the measurement area was $-0.115<X / c<3.366$ and $-0.395<Y / c<0.413$. Three-component measurements were carried out using stereoscopic PIV in the $(X, Y)$ plane in order to check the magnitude of the $w$ component and Reynolds stress $\overline{w^{\prime 2}}$ in the presence of the longitudinal vortex. The two cameras are mounted in the forward scatter direction at an angle of $45^{\circ}$ to the laser sheet plane. A Scheimpflug adapter ensures that particles within the light sheet are in focus across the entire measurement area. The calibration of the PIV system is done using a 3-D calibration plate. The calculation of the velocity vectors is carried out using Dynamic Studio software (Dantec Dynamics). In order to obtain a good spatial accuracy and a minimization of spurious data, the velocity vectors are calculated iteratively using window deformation. The process starts with an interrogation area of $32 \times 32$ pixels and, after four iterations, convergence is reached with a final interrogation area of $16 \times 16$ pixels with $50 \%$ overlap. This yields a spatial resolution of $1.7 \mathrm{~mm}$ between two neighboring vectors. For the temporal resolution of the PIV technique the data rate was $1000 \mathrm{~Hz}$ for 3000 pairs of images corresponding to a $3 \mathrm{~s}$ signal for a characteristic shedding frequency around $30 \mathrm{~Hz}$. Each plane is composed of $77 \times 48$ vectors and a thousand pairs of images were analyzed to generate converged flow field statistics. The convergence of the standard deviation of the velocity is reached for over 500 images. Thus, the results corresponding to 1000 images per each PIV plane provide fully converged turbulence statistics. Additionally convergence was reached for times greater than 
(a)

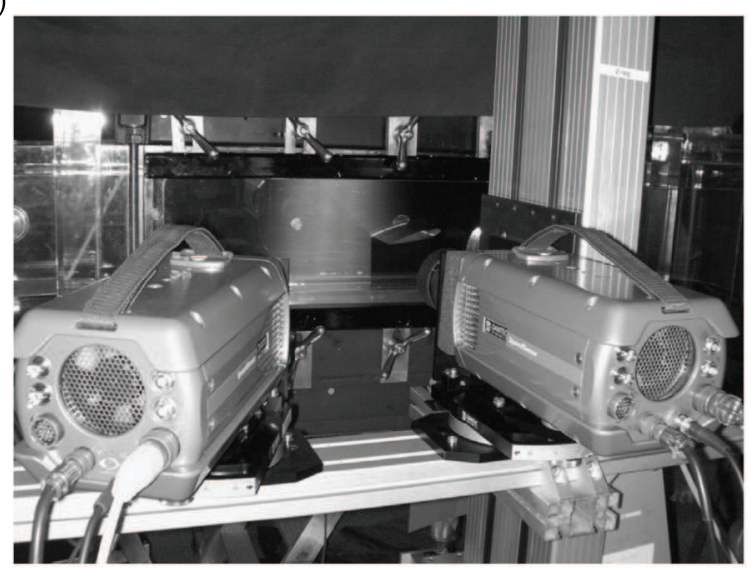

(c)

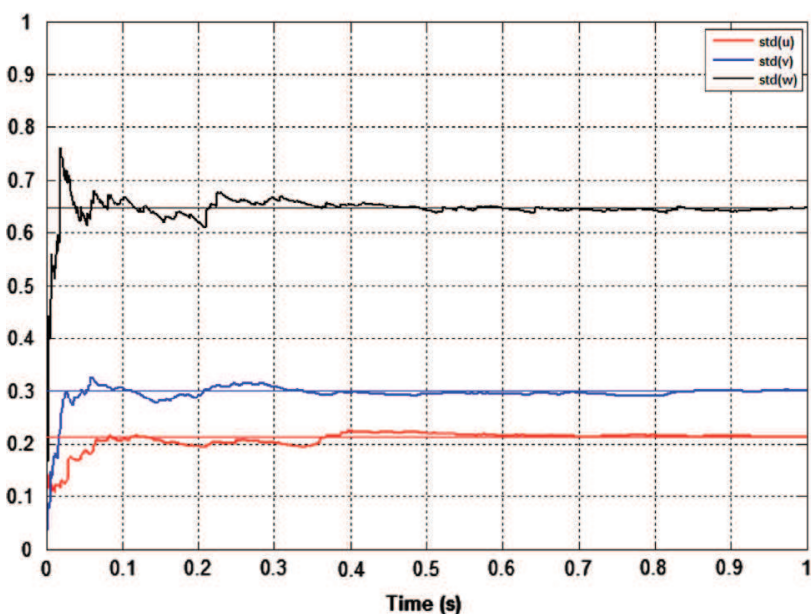

(b)

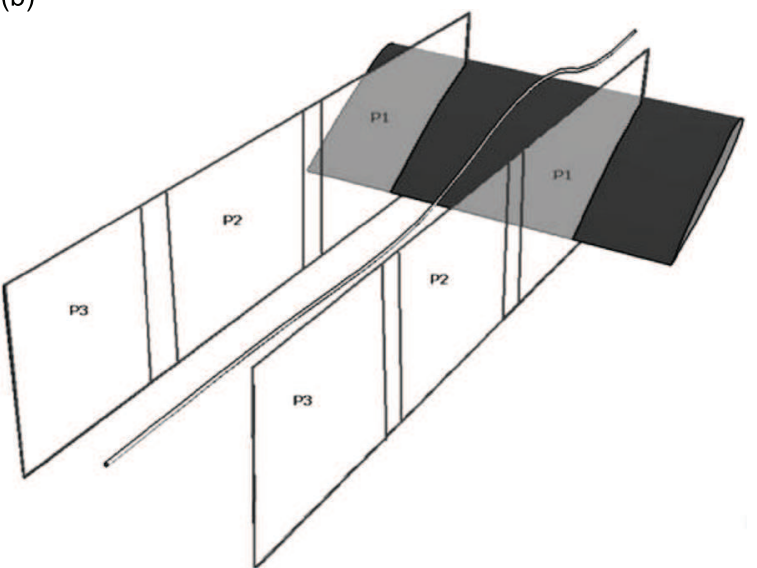

(d)

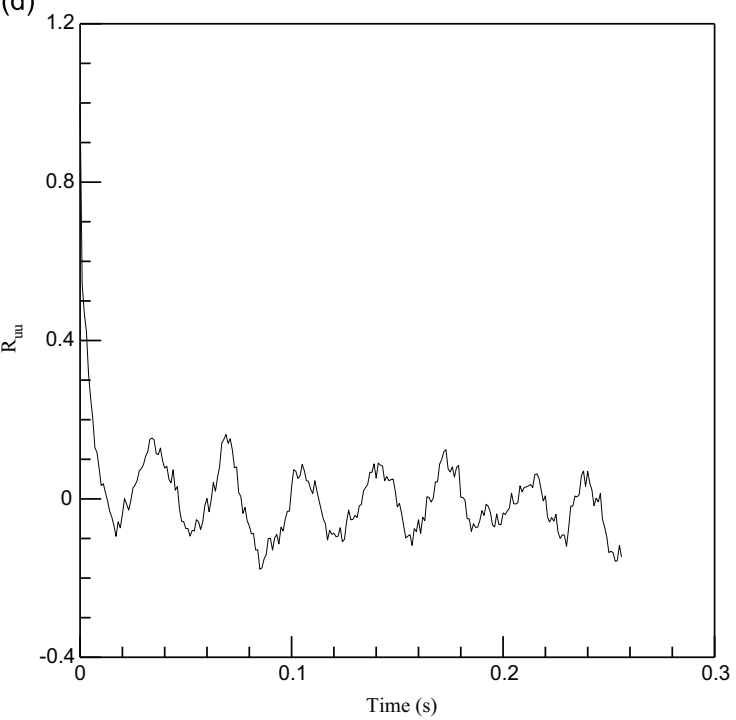

Fig. 2. (a) PIV set-up, (b) configuration of measurement planes, (c) statistical convergence test for the PIV measurement: rms values versus time and (d) autocorrelation function versus time.

$0.1 \mathrm{~s}$ for the standard deviation values of the velocity as shown in Fig. 2(c). All results presented in the present paper were taken for a measurement time of $1 \mathrm{~s}$. This corresponds to an operating process of camera and laser at a frequency of $1000 \mathrm{~Hz}$ and acquisition rate of 1000 images. The correlation function was calculated and is presented in Fig. 2(d). The calculated temporal integral time scale is $7 \mathrm{~ms}$ for an observation time of $1 \mathrm{~s}$. This sample rate was sufficient to observe the physical mechanism of the present configuration of the flow motion.

\subsection{Force measurements}

The lift and drag measurements were performed using a resistive gauge hydrodynamic balance. The maximum ranges were $0-1800 \mathrm{~N}$ for the lift force and $0-170 \mathrm{~N}$ for the drag force. The mean and root mean square $(\mathrm{rms})$ values were calculated from $30 \mathrm{~s}$ test measurements carried out at $2048 \mathrm{~Hz}$. The determination of the hydrodynamic parameters was performed for incidence angles in the range of $\left[-20^{\circ}, 30^{\circ}\right]$.

\section{Effects of the longitudinal vortex on the velocity fields}

\subsection{Inhibition of the hysteresis behavior of forces}

Classical behavior of both lift and drag coefficients (respectively, noted $C_{l}$ and $C_{d}$ ) is observed for low incidence angles (see Fig. 3(a) and (b)) for both reference conditions and with vortex. In Particular, for low incidences, a linear behavior of the 
(a)

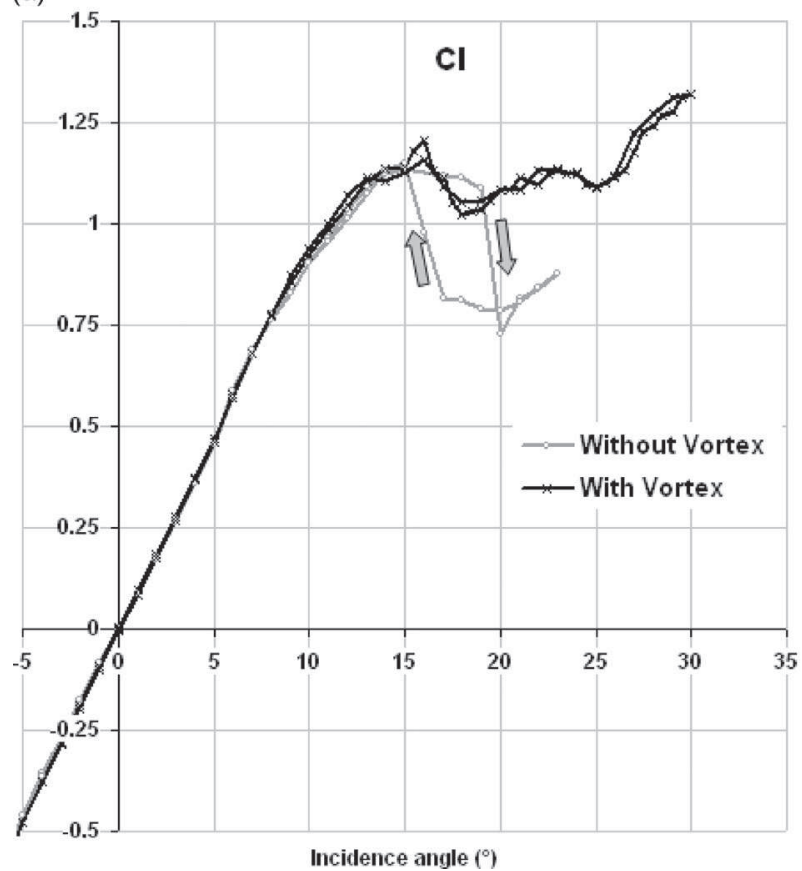

(b)

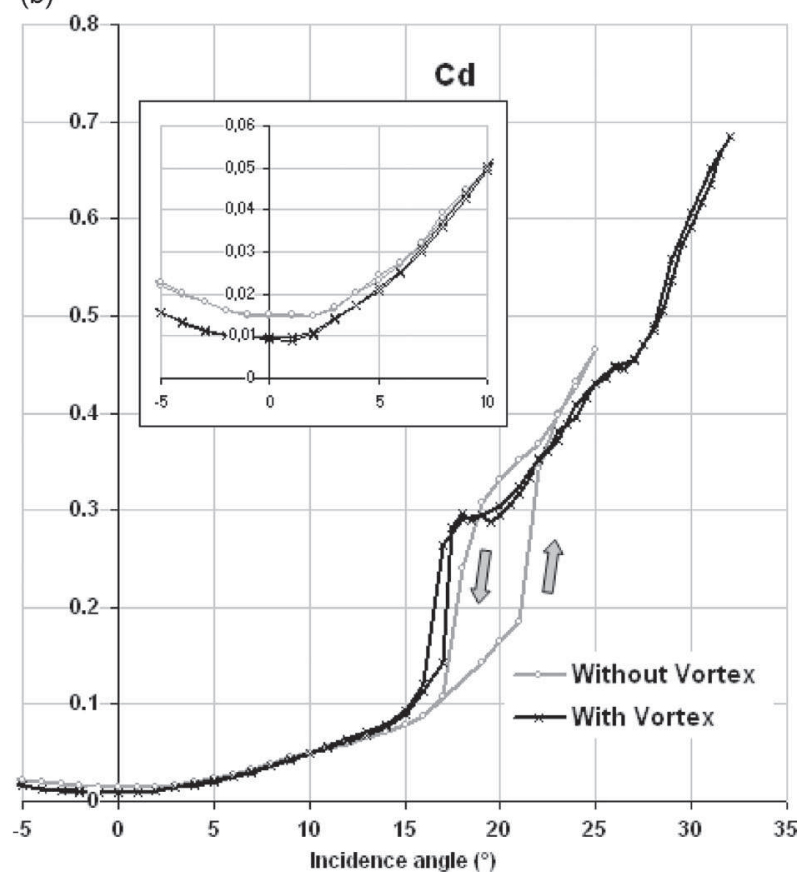

Fig. 3. mean values of global coefficient versus incidence angle: (a) lift coefficient $C_{l}$ and (b) drag coefficient $C_{d}$.

lift, in the range $0-7^{\circ}$, and a quadratic evolution of the drag is observed. The trends are similar with or without vortex and the lift values are not modified by the presence of the vortex.

A modification is observed on the drag coefficient at low incidences (see inset Fig. 3(b)) which is $30 \%$ smaller in the presence of the upstream vortex. In the reference conditions an abrupt loss of lift, which is characteristic of static stall, is observed at an incidence angle of about $17^{\circ}$, associated with an increase in the drag coefficient. This behavior is characteristic of a hysteresis loop install behavior at lower Reynolds numbers $\left(10^{3}<\operatorname{Re}<10^{4}\right)$. Additionally, the lift and drag data for the static foil are collected by increasing the angle of incidence in very small increments.

Concerning the hysteresis loop at static stall, this phenomenon has been observed previously by Hoffmann (1991) and by Mittal and Saxena (2000). Furthermore, Sarraf et al. (2010) studied the thickness effect of NACA foils on hydrodynamic global parameters (e.g. $C_{l}$ and $C_{d}$ ), boundary layer characteristics and stall establishment. In that paper the authors highlighted the hysteresis loop of the lift and drag coefficients. This is indicated in Fig. 3(a) and (b). The starting point for that flow along the increasing angle branch (upper branch) is a partially detached flow in which the detachment point progressively moves from the trailing edge to the leading edge. On the decreasing angle branch (lower branch), a strongly separated flow was observed. The corresponding boundary layer pattern was described by Sarraf et al. (2010) in detail as well as the vortex shedding process via the spectral analysis. This hysteresis is due to an oscillation of the detachment point affected by the Kelvin Helmholtz instability in the shear layer. The nonlinear interaction between the von Kàrmàn vortex shedding from the trailing edge and Kelvin Helmholtz vortices (in the shear layer) appear to be responsible for the coexistence of the two states of the flow (partially and strongly detached). The present experiment for the lift and drag measurements was carried out in the same test section by changing the incidence angle step by step giving a static configuration to the measurements showing very good repeatability of the mean and rms values of the forces.

At high incidences the presence of the vortex inhibits hysteresis and a plateau value is observed associated with an increase in lift of about $20 \%$. The drag coefficient exhibits a specific behavior corresponding to fully detached flow but without hysteresis. The hysteresis loop has been associated with a modification of the position of the separation point. It is to be supposed that the presence of the vortex influences its behavior and inhibits its oscillations. The rms values of the lift and drag coefficients are plotted in Fig. 4(a) and (b). For the lift coefficient, the rms values in the two configurations behave similarly. For the drag coefficient an increase in the rms value is observed and is slightly larger in presence of the vortex. The main difference is observed beyond $15^{\circ}$, where the value is higher than the one obtained without vortex interaction. For this reason the coherent and turbulent process will be analyzed for detachment value over $25^{\circ}$, where the flow is strongly separated.

\subsection{Reynolds-averaged flow fields}

As observed by Sarraf et al. (2010), at high incidence, the near wake of the NACA0015 profile is classically characterized by the formation of organized motion. The topology of the mean and turbulent flows exhibits a pattern of two symmetric 
(a)

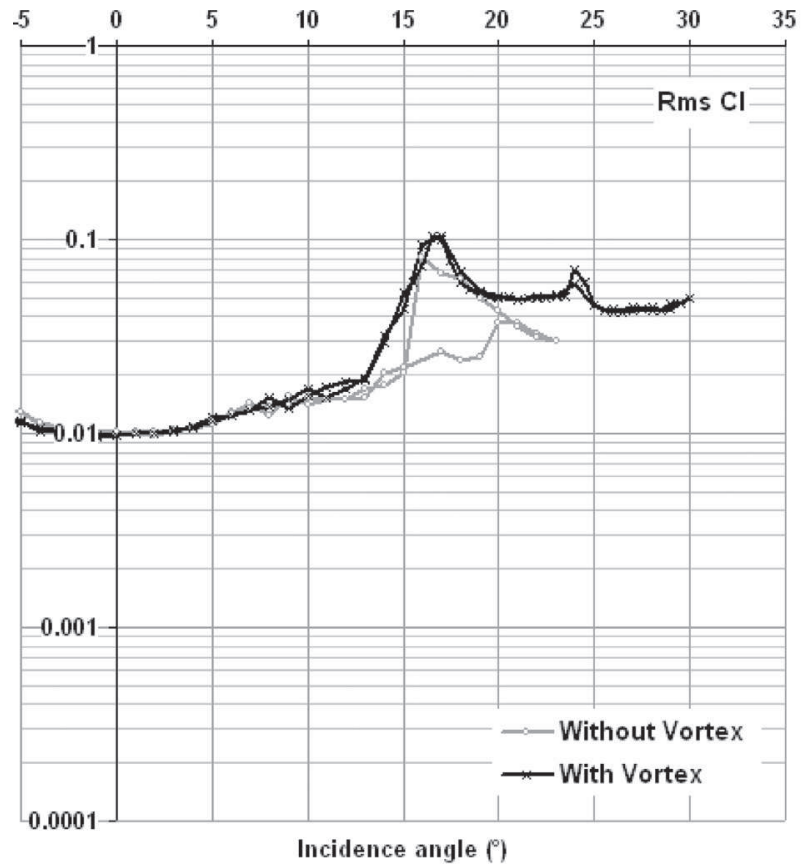

(b)

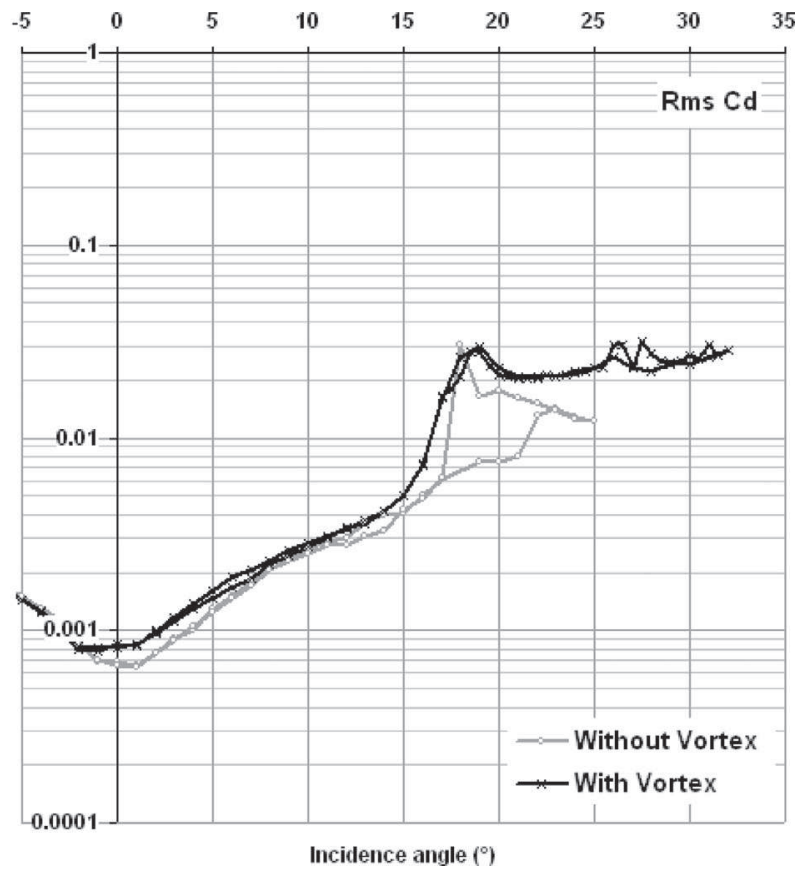

Fig. 4. rms Values of global coefficient versus incidence angle: (a) rms values of lift coefficient $C_{l}$ and (b) rms values of drag coefficient $C_{d}$.

(a)

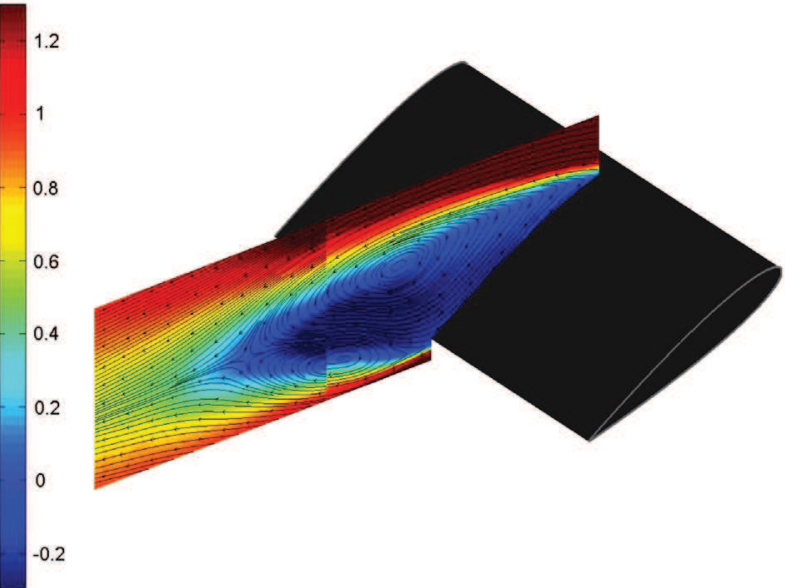

(b)

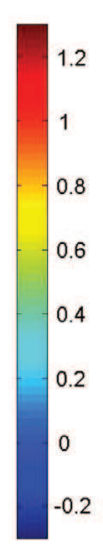

Fig. 5. Iso-contours of normalized mean longitudinal velocity $U$ in the $(x, y)$ plane at $25^{\circ}$ : (a) without upstream vortex and (b) with upstream vortex. Inflow and outflow areas are presented.

eddies which is due to time averaging the passage of the alternating vortices from the leading and trailing edges. The iso- $U$, iso- $V$ and iso- $W$ contours and streamlines without interaction are shown in Figs. 5(a,b), 6(a,b) 7(a,b) respectively and compared to those obtained in the two characteristic areas inflow and outflow associated with the presence of the upstream longitudinal vortex. The mean iso- $V$ contours (see Fig. 6(a,b)) illustrate the anti-symmetric character of this component due to the passage of the alternating eddies. Concerning the iso- $W$ contours (see Fig. $7(\mathrm{a}, \mathrm{b})$ ), the almost zero value illustrates the 2-D pattern of the mean components.

The above measurements allow the determination of the location the saddle points, the center of the leading and trailing edge vortices in the near wake, and the formation length $l_{U}$ (based on the location of the minimum axial velocity) and recirculation length $l_{C}$ (given for the saddle point). These different parameters are given in Table 1 . In the presence of the upstream vortex, the interaction is characterized by inhibition of the vortex shedding in the inflow area. In the outflow area we observed a change in the saddle point location where the vortex induces a velocity directed from the wall to undisturbed flow. The major change corresponds to the longitudinal and vertical movement of the trailing and leading edge vortex cores 
(a)

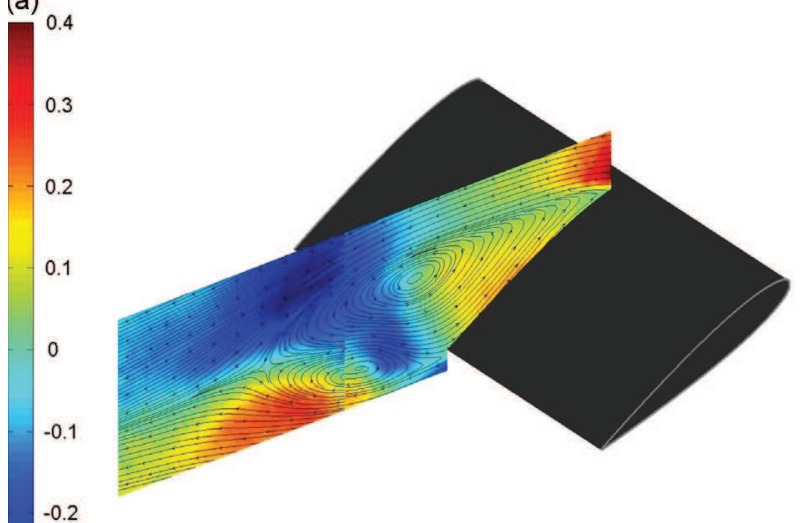

(b)

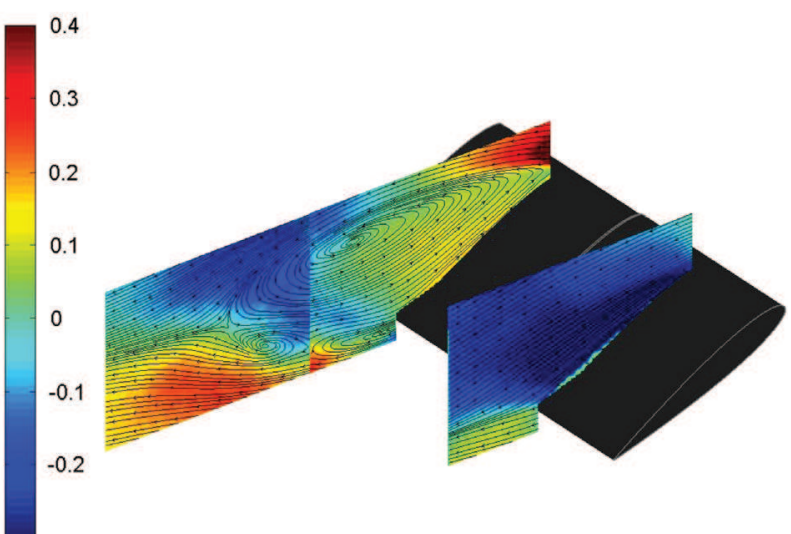

Fig. 6. Iso-contours of normalized mean vertical velocity $V$ in the $(x, y)$ plane at $25^{\circ}$ : (a) without upstream vortex and (b) with upstream vortex. Inflow and outflow areas are presented.

(a)

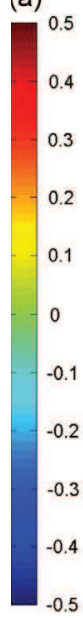

(b)

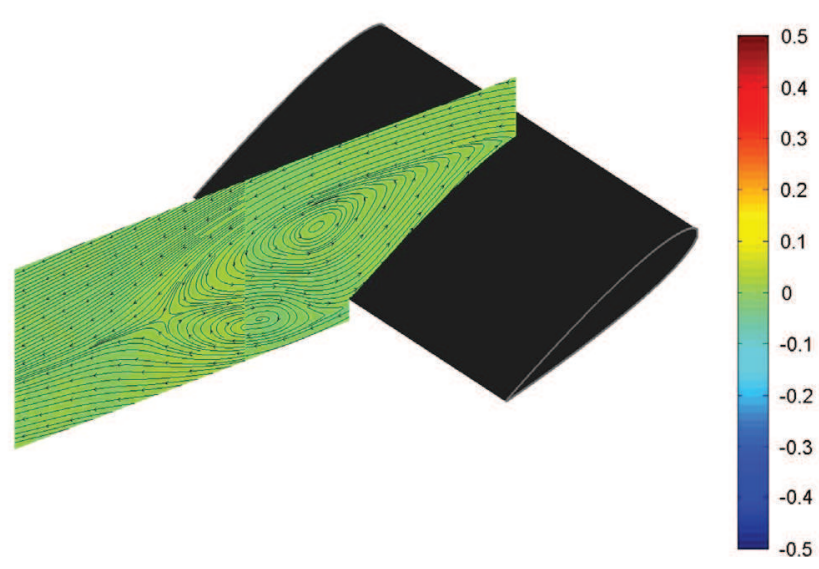

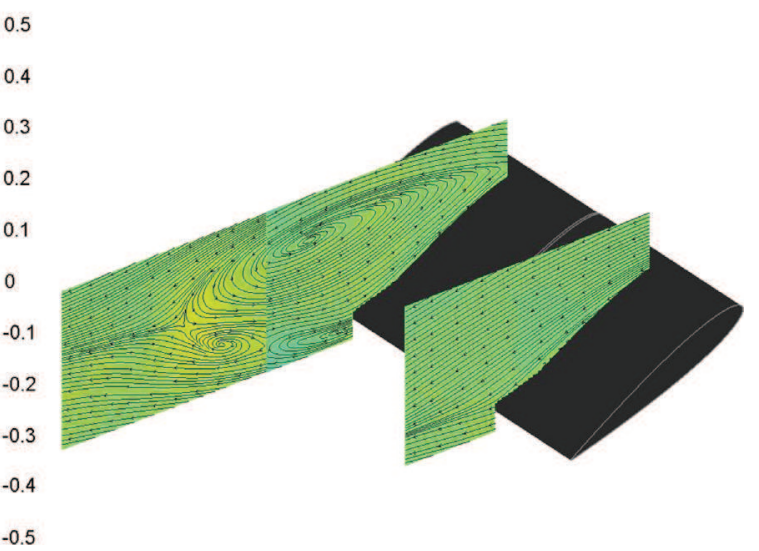

Fig. 7. Iso-contours of normalized mean spanwise velocity $W$ in the $(x, y)$ plane at $25^{\circ}$ : (a) without upstream vortex and (b) with upstream vortex. Inflow and outflow areas are presented.

Table 1

Locations of the vortex cores and characteristic length values with and without vortex.

\begin{tabular}{llll}
\hline Characteristics positions & Without vortex & \multicolumn{2}{l}{ With vortex } \\
\cline { 3 - 4 } & & Inflow & Outflow \\
\hline Leading edge & $X / c=0.837$ & - & $X / c=0.954$ \\
Vortex core & $Y / c=0.054$ & & $Y / c=-0.169$ \\
Trailing edge & $X / c=1.107$ & $X / c=1.413$ \\
Vortex core & $Y / c=-0.253$ & & $Y / c=-0.168$ \\
Recirculation length lc & $X / c=1.546$ & - & $X / c=1.592$ \\
& $Y / c=-0.099$ & & $Y / c=-0.037$ \\
Formation length lu & $X / c=1.080$ & - & $X / c=1.116$ \\
& $Y / c=-0.123$ & & $Y / c=-0.093$ \\
\hline
\end{tabular}

respectively. Interaction in the outflow area is characterized by an expansion of the wake in the downstream direction, which is associated with a thickening effect in the vertical direction as observed on the iso- $U$ contours. The vortex transport is associated with the inhibition of the hysteresis at static stall. Furthermore, the presence of the vortex does not induce a transverse mean motion in the outflow region and the flow remains two-dimensional. There is a slight increase in the formation length associated with the appearance of a vortex downstream of the trailing edge in the presence of the upstream tip vortex. 
The backflow coefficient was used in order to ascertain the effect of the upstream vortex. The definition of this coefficient, called $\chi$ (sometimes written as $\gamma_{p u}$ ) is the ratio between the time during which the flow as a negative velocity component (along the $x$ axis) to the total time considered. This number is defined as follows:

$$
\chi=\frac{T_{U<0}}{T_{\text {total }}}
$$

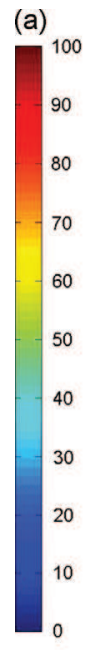

(b)
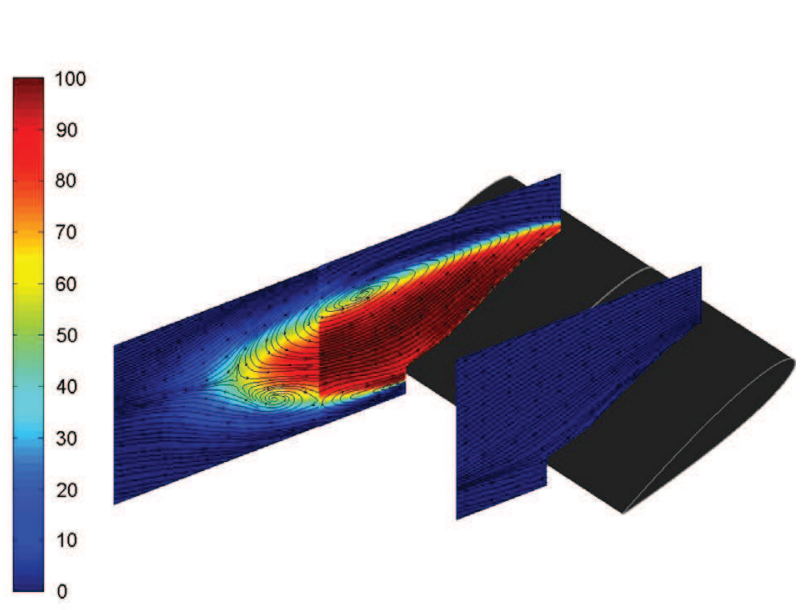

(c)

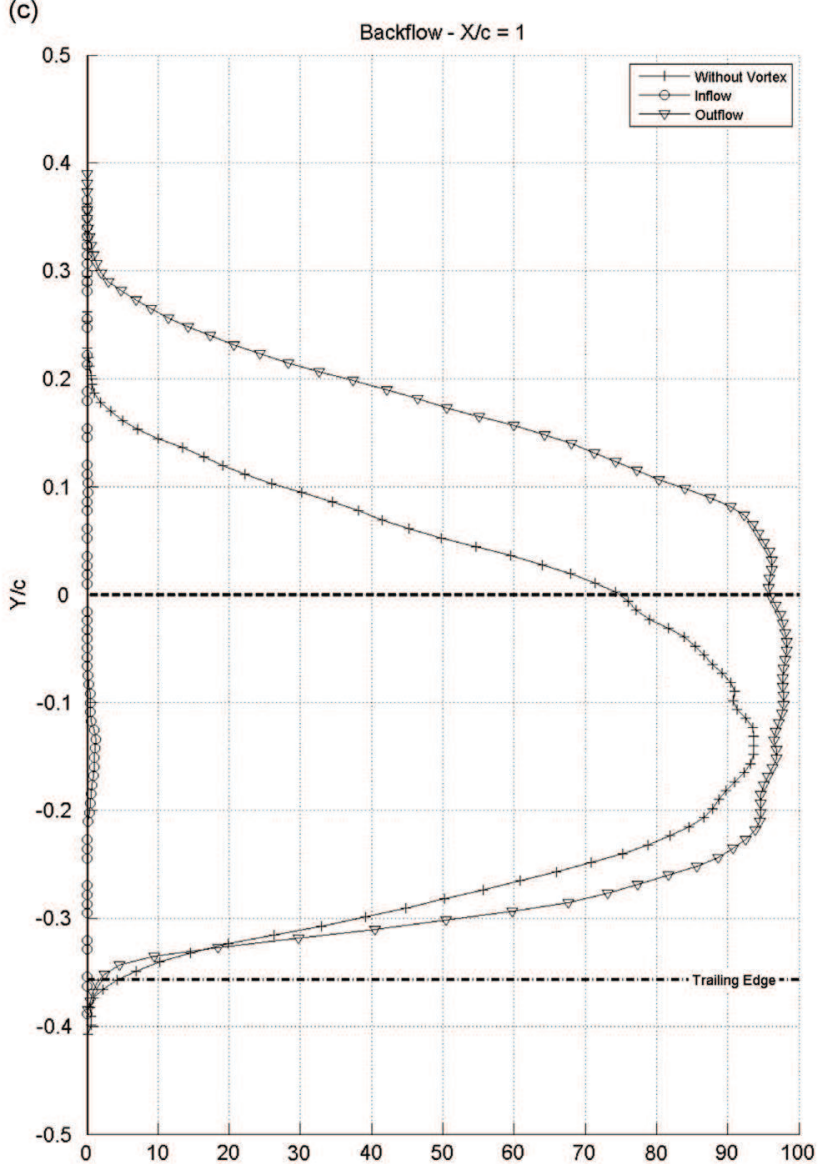

Fig. 8. Iso-contours of the backflow coefficient: (a) without upstream vortex, (b) with upstream vortex and (c) extracted profile of the backflow coefficient at $x / c \quad 1$. 
Most investigations indicate that the 0.5 value coincides with the point of vanishing wall shear stress (Simpson, 1989). Iso- $\chi$ contours shown in Fig. $8(\mathrm{a}-\mathrm{c})$ shows the extracted profile at $x / c=1$. The main observed difference, when the upstream vortex is present, consists in the more extended backflow in the outflow region while, in the inflow area, the flow is locally reattached due to high momentum fluid displacement. This demonstrates the effectiveness of the tip vortex to modify the separated area even if it does not impact against the NACA0015 foil.

Measurement of the mean velocity components allows the quantification of velocity gradients in the near region, at different positions in the spanwise direction. These velocity gradients were evaluated with central difference discretization. In this way, the rotation rate tensor or vorticity component $\omega_{12}=(1 / 2)((\partial \bar{V} / \partial x)-(\partial \bar{U} / \partial y))$ was determined and the graphical representation is presented in Fig. 9(a) and (b). As expected, without interaction the vorticity field exhibits an antisymmetric aspect corresponding to the formation of two shear layers: one from the leading edge and the other from the trailing edge in which the highest values are found near the detachment point. In the presence of the upstream tip vortex, the iso-vorticity contours are quite similar to the unperturbed case in the outflow area except for a slight difference in the trailing edge vortex which is somewhat longer in the near wake. The major change can be seen for the inflow region where the two shear layers (corresponding to vortex shedding) disappear.

Normal turbulent quantities and Reynolds stresses fields $\sqrt{\overline{u^{\prime} v^{\prime}}} / U_{\text {inf }}$ are quantified with and without upstream vortex and the topology of the near wake consists of two-lobe and one-lobe structures for the longitudinal and vertical components respectively. A global decrease in normal quantities was observed, of approximately $3 \%$ for $\sqrt{\overline{u^{\prime 2}}} / U_{\text {inf }}$ and $25 \%$ for $\sqrt{\overline{v^{\prime 2}}} / U_{i n f}$. The Reynolds stresses take the largest values on either side of the wake center-line (presented in Fig. 10(a,b)). In the inflow area an inhibition of the double peak-shape (due to the alternating vortices in the wake) and an increase of

(a)

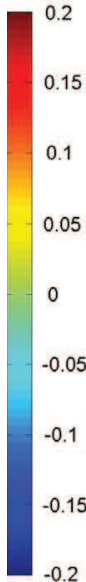

15

(b)

(a)

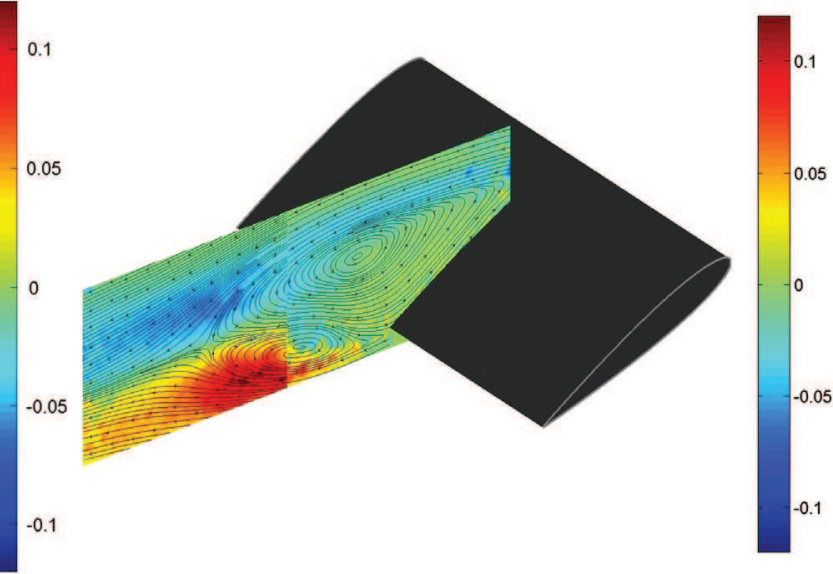

(b)
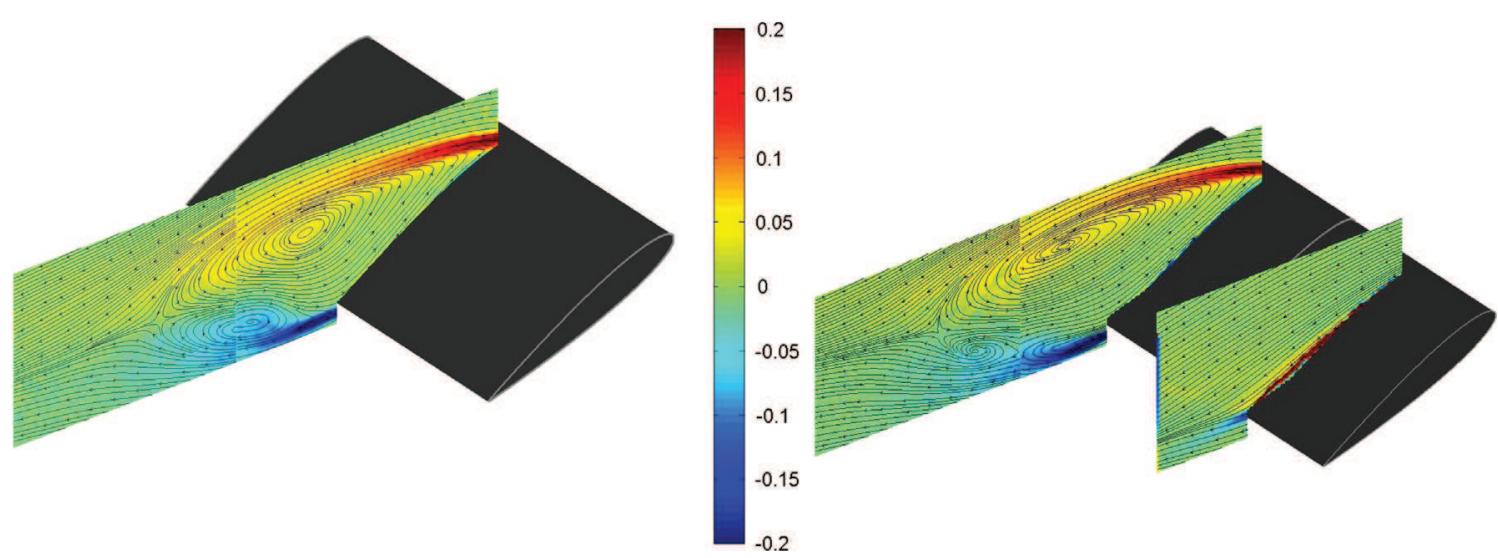

Fig. 9. Iso-contours of normalized vorticity $\omega_{\mathbf{1 2}}$ : (a) without upstream vortex and (b) with upstream vortex.

Fig. 10. Iso-contours of Reynolds stresses $\overline{u^{\prime} v^{\prime}}$ : (a) without upstream vortex and (b) with upstream vortex. 
$22 \%$ for $\sqrt{\overline{u^{\prime} v^{\prime}}} / U_{\text {inf }}$ is observed. The maximum values are localized at $y / c=-0.25$ for outflow instead of -0.3 without interaction. This is due to the mean motion and the dissymmetry of the trailing and leading edge vortices. The same trend was observed by Angele and Muhammad-Klingmann (2005) for the case of streamwise vortex interaction with a separated turbulent boundary layer. The authors found a significant decrease in the level of streamwise fluctuations. Fig. 11(a) and (b) shows the two dimensional aspect of the wake with and without interaction. The stereoscopic measurements allow the determination of the turbulent kinetic energy $k=(1 / 2)\left(\overline{u^{\prime 2}}+\overline{v^{\prime 2}}+\overline{w^{\prime 2}}\right)$ without any assumption made concerning the third normal stress. The iso-contours of turbulent kinetic energy are shown in Fig. 12(a) and (b) with and without vortex interaction. The fluctuation levels are evident via the color contours, while the streamlines have been superimposed to indicate the centers of vortices and the saddle points in the formation region. Without interaction, turbulent kinetic energy exhibits a one-lobe structure with a maximum located at $x / c=1.18$ and $y / c=-0.29$ near the trailing edge. The maximum decreased by $23 \%$ and is located at $x / c=1.33$ and $y / c=-0.26$ in the outflow area corresponding to the mean vertical motion of the trailing edge vortices. In the inflow area, there is inhibition of the one-lobe structure and a global decrease in turbulent activity for all quantities. This trend corroborates the previously observed decrease in the global production of fluctuations. Concerning the turbulent production term $P=-\overline{u_{i}^{\prime} u_{j}^{\prime}}\left(\partial \overline{U_{i}} / \partial x_{j}\right)$, shown in Fig. 13(a,b), the maximum values are located in the shear layer near the separation point without interaction at $x / c=1.25$ and $y / c=-0.29$. The turbulent production is more extended in the outflow region in the presence of the longitudinal vortex (in outflow region: $x / c=1.28$ and $y / c=-0.31$ ). In the inflow area, the production is inhibited in the shear layer because of the vortex shedding process.

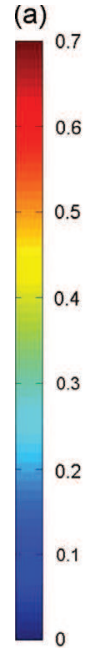

(a)

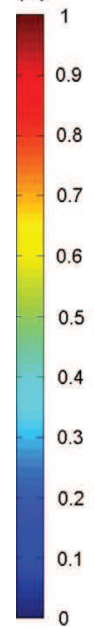

(b)

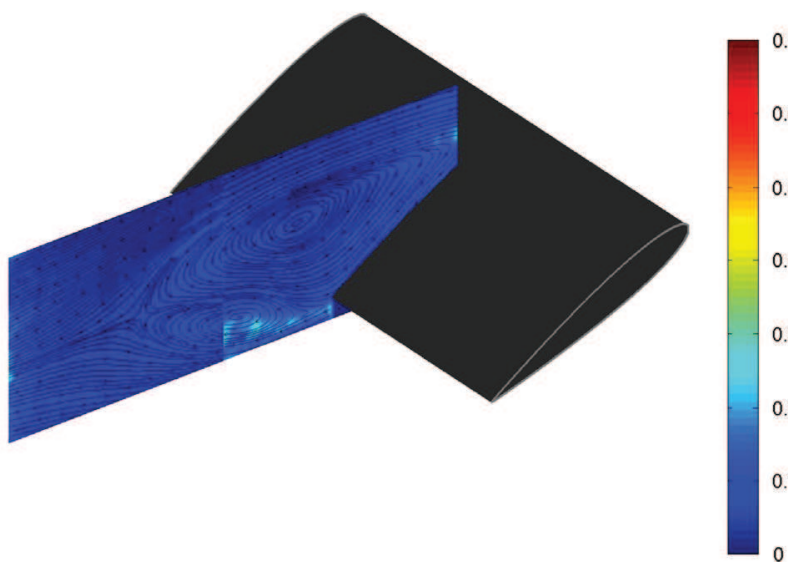

Fig. 11. Iso-contours of spanwise fluctuations $\sqrt{\overline{w^{\prime 2}}}$ : (a) without upstream vortex and (b) with upstream vortex.
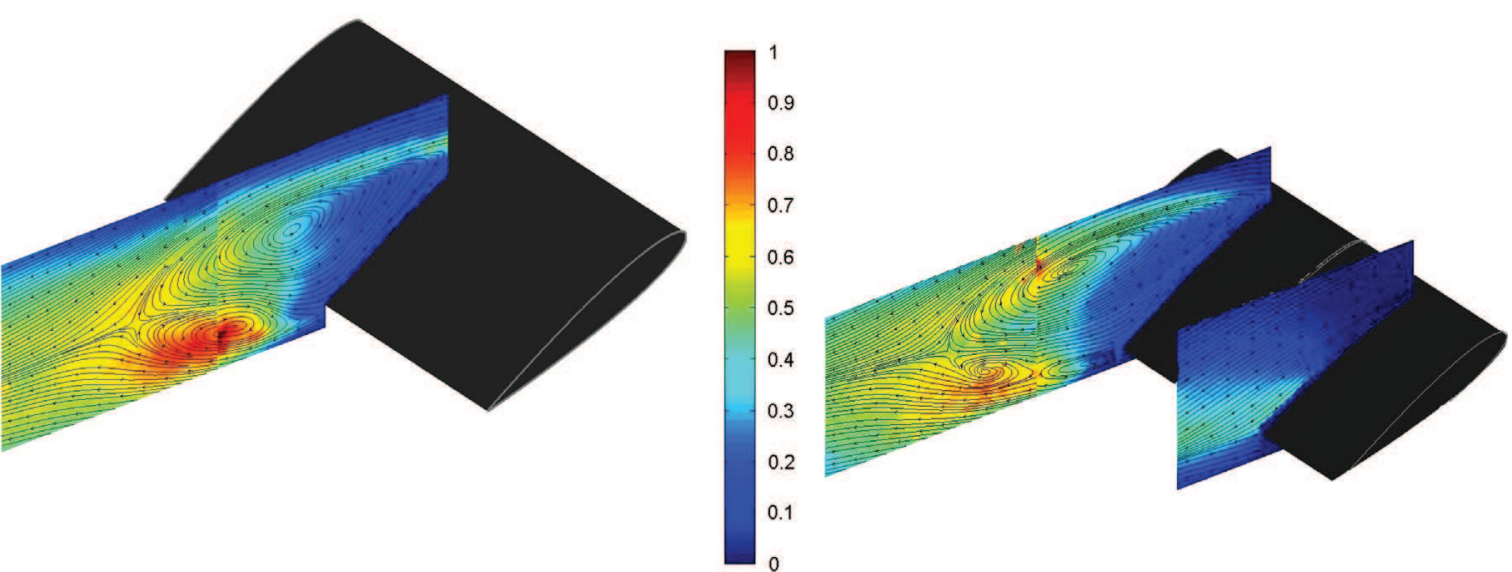

Fig. 12. Iso-contours of the turbulent kinetic energy $k$ : (a) without upstream vortex and (b) with upstream vortex. 
(a)

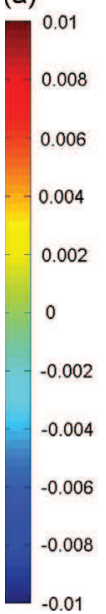

(b)

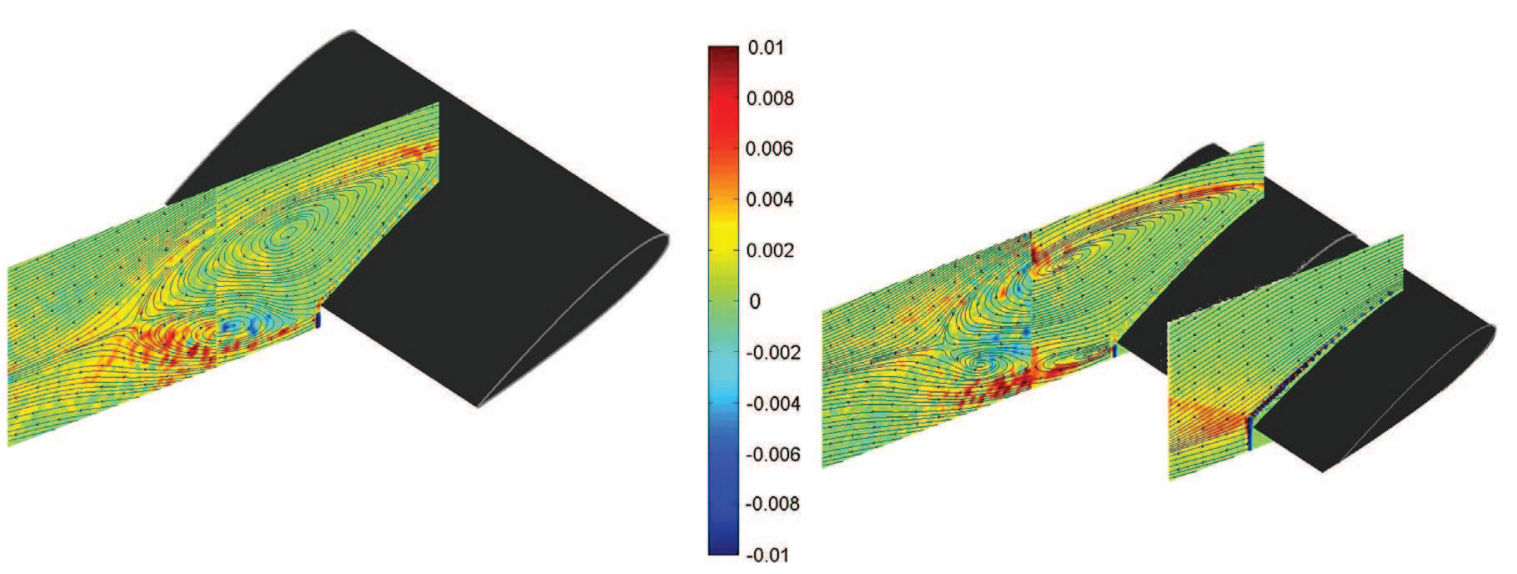

Fig. 13. Iso-contours of the turbulent production $P$ : (a) without upstream vortex and (b) with upstream vortex.

\section{POD analysis and phase averaging for the coherent process}

\subsection{Method description and results}

The Proper Orthogonal Decomposition (POD) also known as the Karhunen Loeve expansion was used to study the coherent structures in the near wake of the foil. In this section only a brief description is presented and more details can be found in the work of Berkooz et al. (1993), for example. The POD analysis consists in searching for the function that is most similar to the members of a data set. This is done by solving an eigen-value problem where the kernel is the two point correlation tensor. The eigenvectors of this problem are the modes $\Phi_{i}^{(k)}(x)$ and the corresponding eigenvalues $\lambda_{k}$ represent the energy contained in each mode. Based on these modes, the velocity fields can be reconstructed by projecting the instantaneous fields on a restricted number of modes. The reconstructed field can then be written as

$$
u_{i}(x, t)=\sum_{k} a_{k}(t) \Phi_{i}^{(k)}(x), \text { where } a_{k}(t)=\int_{\Omega} u_{i}(x, t) \Phi_{i}^{*(k)}(x) d V .
$$

This previous decomposition is optimal in the sense that any reconstruction using another basis, with the same number of modes contains less energy. The decomposition of the two point correlation tensor can be written as

$$
u_{i} u_{j}(x)=\sum_{k} \lambda_{k} \Phi_{i}^{(k)}(x) \Phi_{j}^{*(k)}(x)
$$

In the present study the snapshot method (Sirovich, 1987) has been used with 3000 instantaneous velocity fields.

The POD method highlights the vortex shedding process with and without interaction. The percentage of the total energy of the fluctuating motion has been investigated in the downstream planes to evaluate the contribution of the two first modes on the shedding process with and without interaction. These distributions are presented in Fig. 14. The fluctuating energy distributions in the domain contained in the 100 first modes in a log-log plot show that the first two modes are of the same order of magnitude. For the P1 plane in the vicinity of the NACA0015 foil, the percentage of the energy of the fluctuating motion decreases rapidly due to the establishment of the von Kàrmàn instability. Downstream, for the $P 2$ and $P 3$ planes the levels are higher and without interaction. The two first modes have quite similar levels of energy, in good agreement with other studies relative to cylinder wake (Perrin et al., 2007). Furthermore no noticeable interactions are observed in the presence of a longitudinal vortex. The energy distribution decreases in the downstream direction, leading to a progressive destruction of the von Kàrmàn instability. The presence of the upstream vortex inhibits flow detachment in the inflow region rendering the energy signature too weak to exploit. In the outflow area for the $P 2$ plane, the shedding process is more energetic than the one observed without interaction leading to a relative contribution of about $30 \%$ instead of $20 \%$ of total energy. Concerning the global representation of each mode, the 0 mode has a quite similar topology to the mean motion and the trailing edge vortex is less pronounced in the outflow area. For the higher order modes (see Figs. 15-18(a,b)), mode 1 comprises a two-lobe counter-rotating structure characteristic of spatial periodic detachment. Mode 2 represents the same structures with a delay in downstream direction of about a quarter wave length. This is in agreement with numerical and experimental studies for various Reynolds numbers in the case of bluff body wake configurations (Noack et al., 2003; Van Oudheusden et al., 2005; Perrin et al., 2007). In the outflow area, a deflection of the symmetric axis of the wake is observed. Note that, for the case without a vortex, the centers of the structures are on a horizontal line, in contrast to the outflow case, where the latter are inclined. Concerning modes 3 and 4, they are responsible for stretching-contraction mechanisms and 

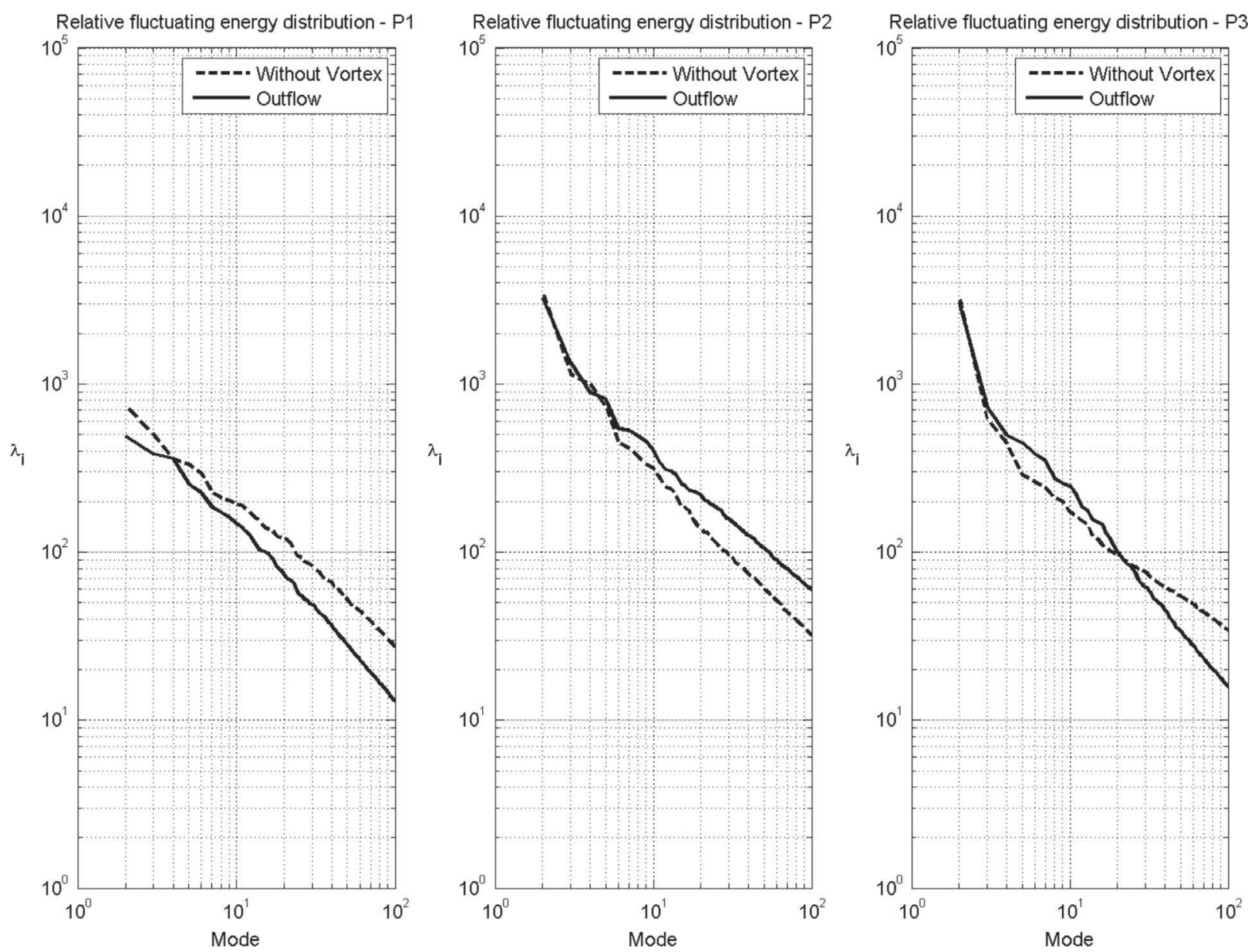

Fig. 14. Fluctuating energy distributions versus modes for the $P 1, P 2$ and $P 3$ planes.

(a)

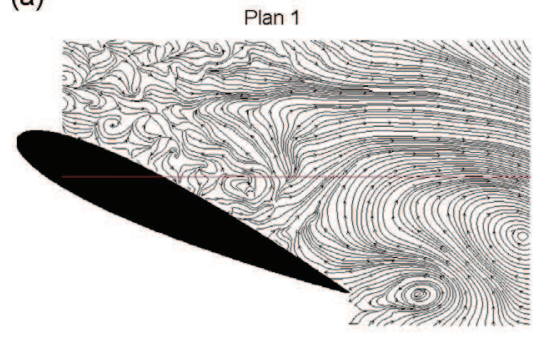

(b)

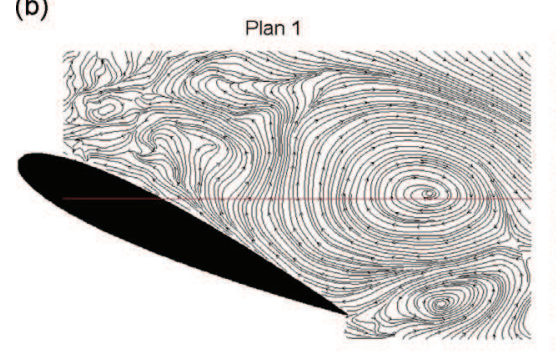

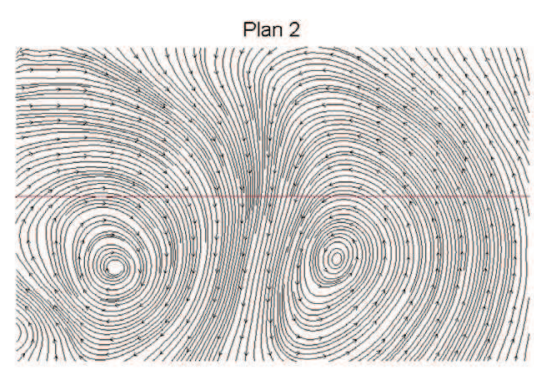

Plan 2

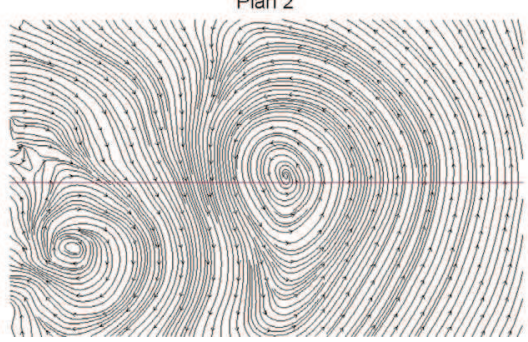

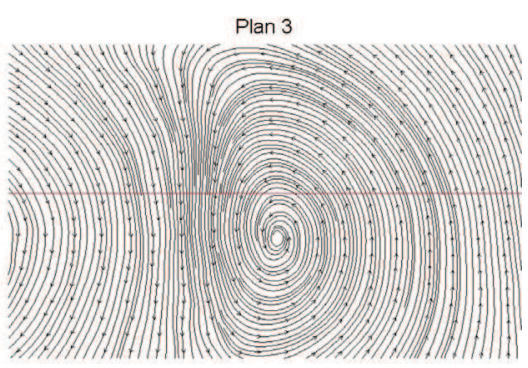

Plan 3

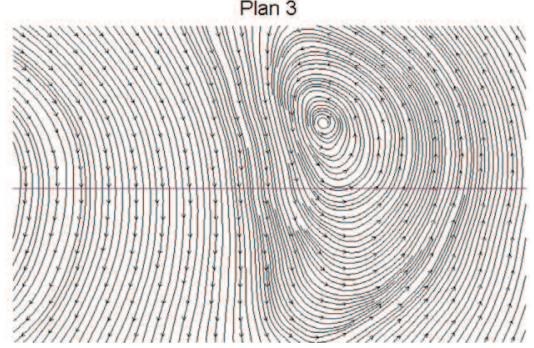

Fig. 15. Representation of mode 1 for the $P 1, P 2$ and $P 3$ planes: (a) without upstream vortex and (b) with upstream vortex. 
(a)

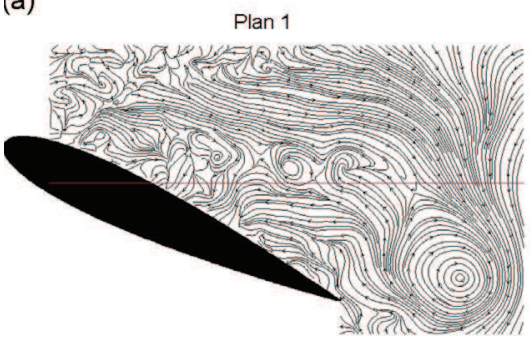

(b)

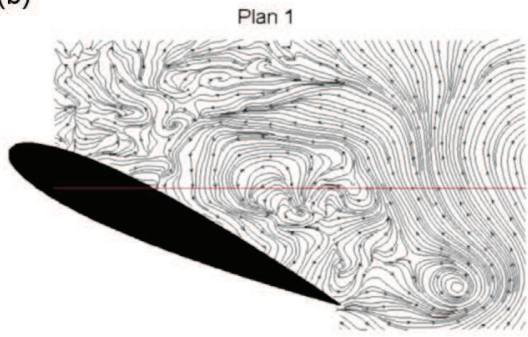

Plan 2

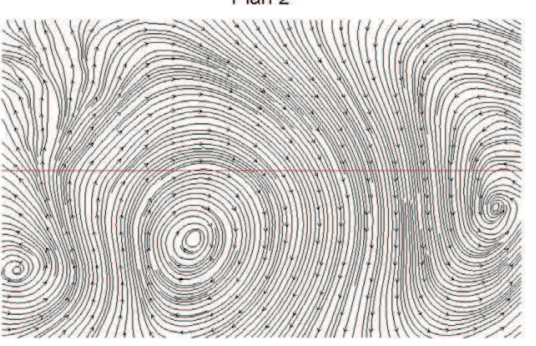

Plan 2

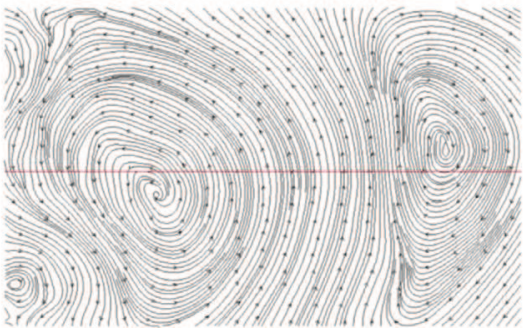

Plan 3

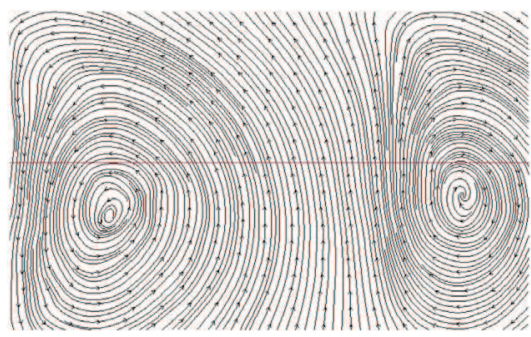

Plan 3

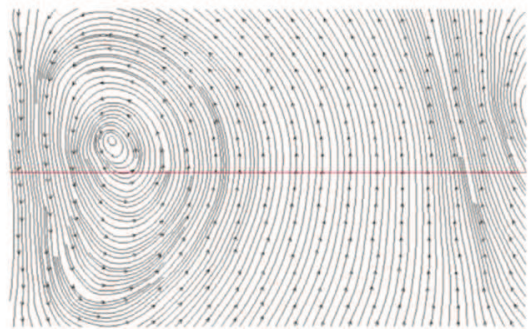

Fig. 16. Representation of mode 2 for the $P 1, P 2$ and $P 3$ planes: (a) without upstream vortex and (b) with upstream vortex.

(a)

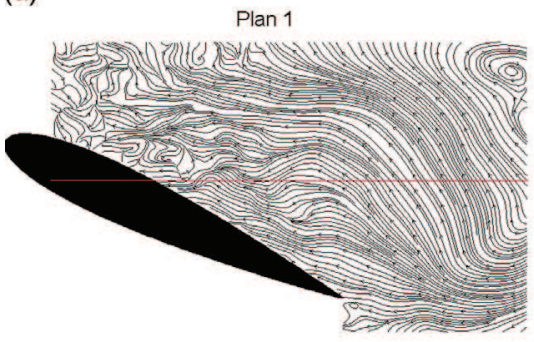

(b)

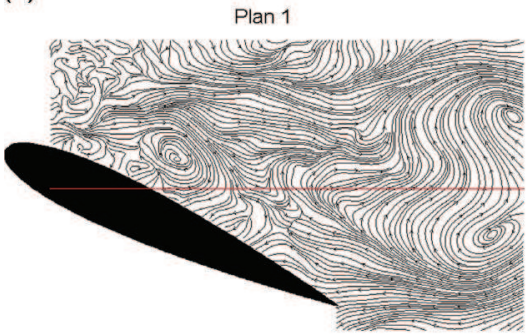

Plan 2

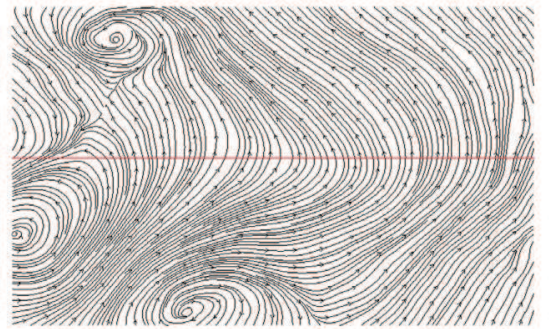

Plan 2

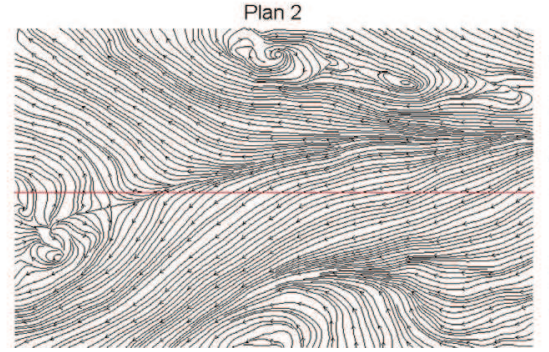

Plan 3

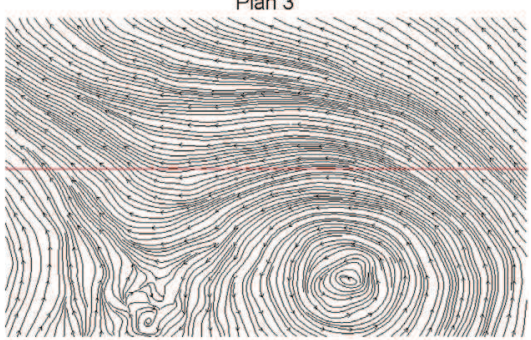

Plan 3

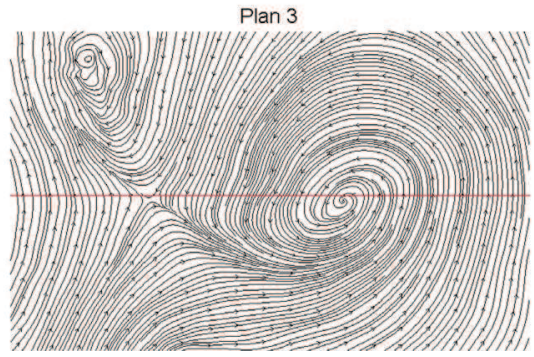

Fig. 17. Representation of mode 3 for the $P 1, P 2$ and $P 3$ planes: (a) without upstream vortex and (b) with upstream vortex.

dissymmetric behavior, respectively. The stretching-contraction phenomenon is modified by the presence of the vortex in the outflow region.

\subsection{Strouhal number}

As noted by Sarraf et al. (2010), once the incidence of the NACA0015 foil increased beyond $20^{\circ}$, organized motion due to the regular vortex shedding became evident and was characterized by a predominant Strouhal number $S_{t}$, close to 0.21 . This number is based on the projected area of the foil and defined as

$$
S_{t}=c \sin \alpha \frac{f}{U} \text {. }
$$

To characterize the Strouhal number, a spectral analysis has been performed on the temporal signal of the longitudinal velocity or on the temporal signals of the first POD coefficients. This spectral analysis was carried out using a Fast Fourier Transform method with a resolution of $\Delta f=0.1 \mathrm{~Hz}$. Typical example of a spectrum is shown in Fig. 19, at $x / c=2.5$ and $y / c=2$ 
(a)

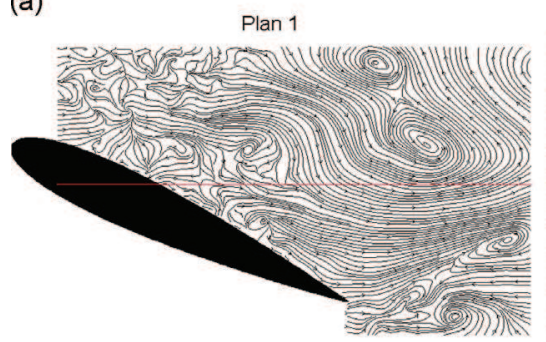

(b)

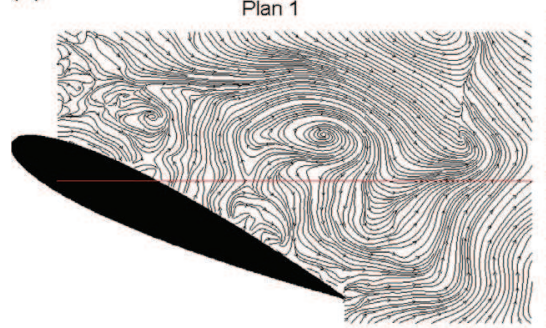

Plan 2

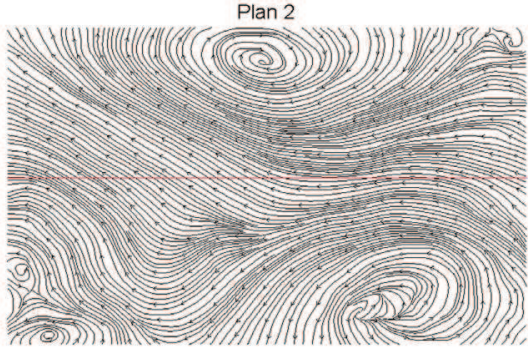

Plan 2

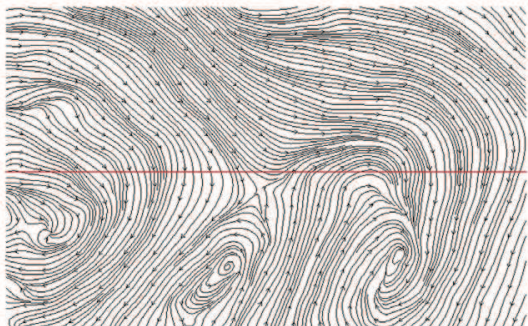

Plan 3

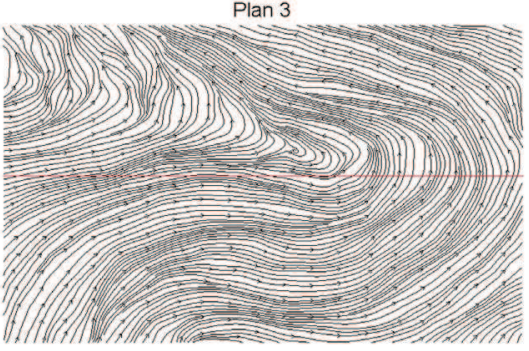

Plan 3

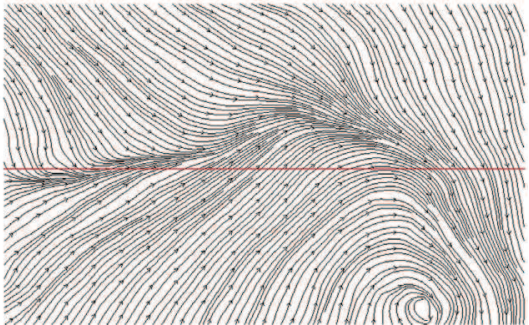

Fig. 18. Representation of mode 4 for the $P 1, P 2$ and $P 3$ planes: (a) without upstream vortex and (b) with upstream vortex.

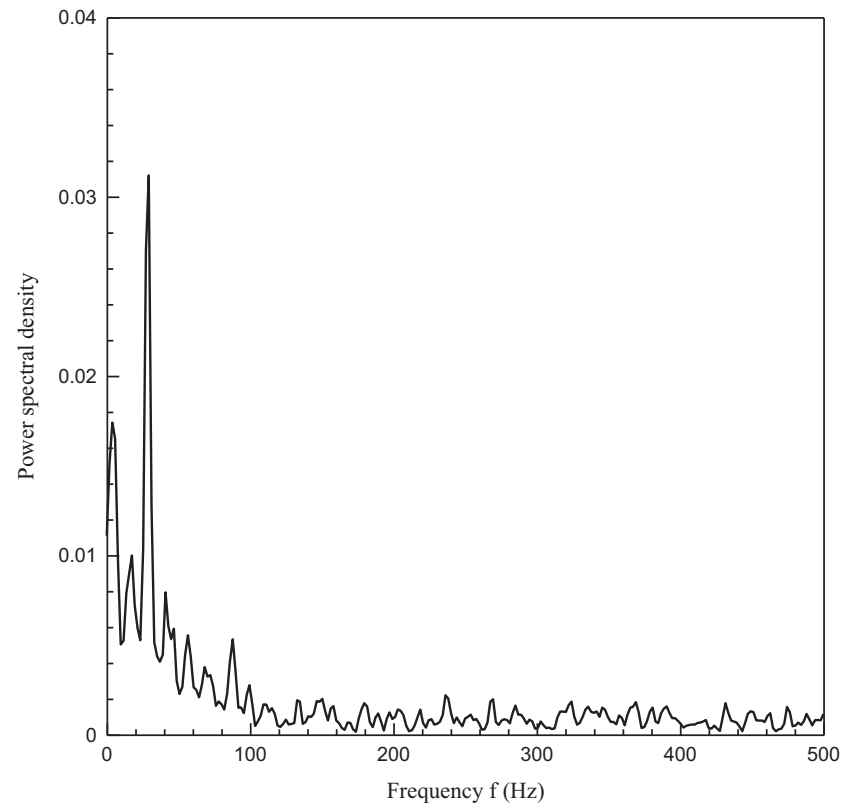

Fig. 19. Example of the spectrum without interactions.

in the configuration without an upstream vortex. The predominant frequency characterizes the organized part of the motion due to the regular vortex shedding. This predominant value corresponds to $30 \mathrm{~Hz}$ (with $S_{t}=0.21$ ).

The decomposition (POD) provides us the temporal coefficient $a_{i}$ associated with mode $\phi_{i}$. This parameter weights each mode in an instantaneous field. There are relationships between the different ranks of decomposition. In the present case, spectral analysis was performed on the temporal signals of the POD coefficients. The two first modes are related to the fundamental frequency of the von Kàrmàn instability. For the rest of the extracted POD modes, the vortex shedding frequency is not clearly identified due to noisy signal and lower energy distributions. In the undisturbed case, the maximum amplitude of the vortex shedding is observed in the shear layer corresponding the maximum vorticity (located at $x / c=1$ ). This value is in good agreement with the development of the von Kàrmàn instability around a circular cylinder at high Reynolds number (Norberg, 2003; Djeridi et al., 2003). In the outflow region the Strouhal number slightly decreases to a value of 0.19 instead of 0.21 , without interaction, in the P3 plane. This trend is the opposite of that observed by Ünal and Atlar (2010) in their study of the effect of a vortex generator on near wake flow around circular cylinder. This change in Strouhal number is due to the modification of the two shear layers and the vortex diffusion. As highlighted by Ünal and Atlar (2010), 
(a)

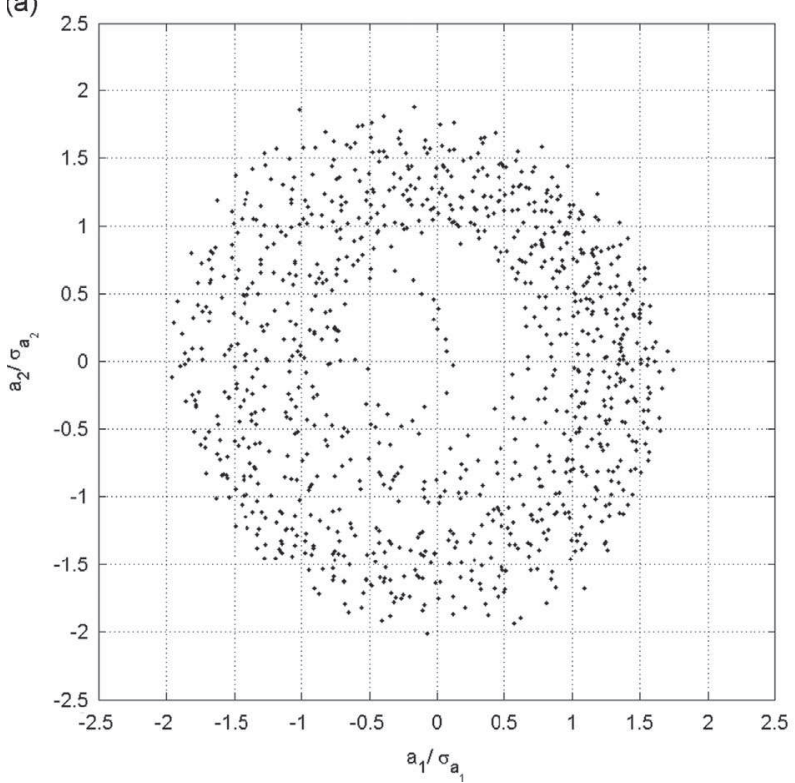

(b)

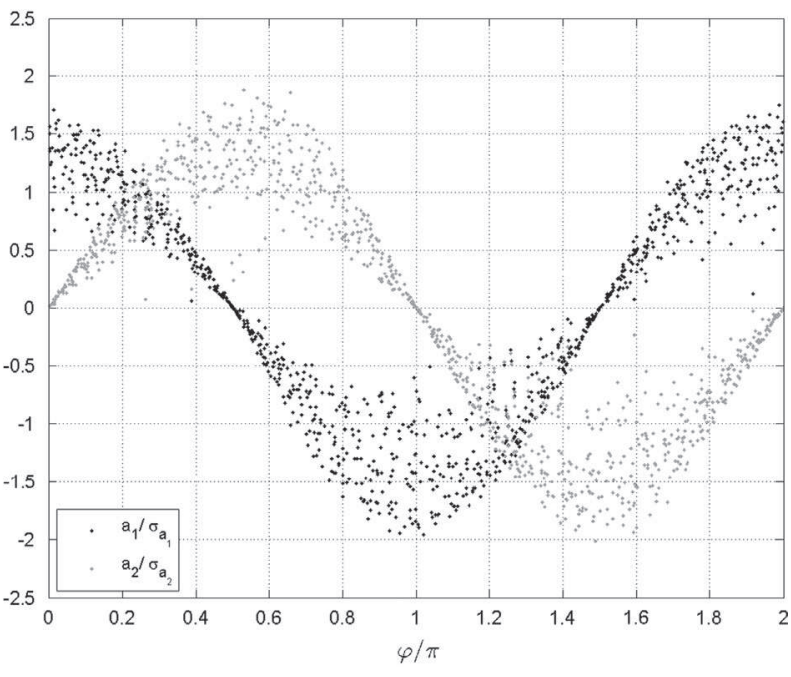

Fig. 20. Representations of the first coefficients for $P 3$ plane and the configuration without vortex: (a) $a_{2}=f\left(a_{1}\right)$, (b) $a_{1}=f(\varphi)$ and $a_{2}=f(\varphi)$.

(a)

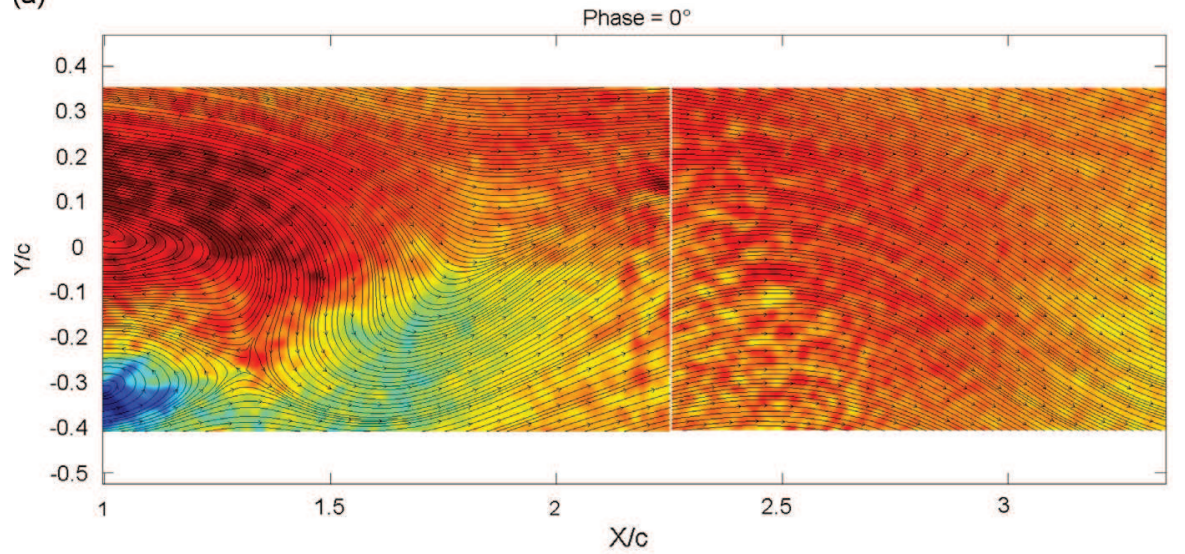

(b)

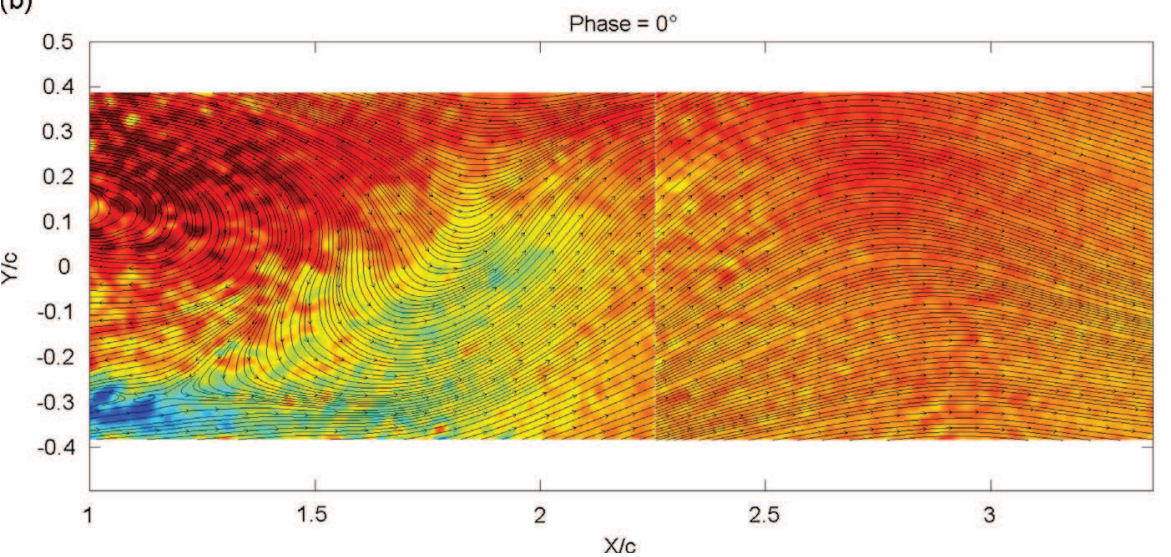

Fig. 21. $0^{\circ}$ Phase averaged vorticity obtained using the POD coefficients: (a) without upstream vortex and (b) with upstream vortex. 
certain factors can affect the fundamental frequency. They noted the change in entrainment in the shear layer and hence also in the turbulent intensity. The location of the shear layer formation and its thickness also changed. In the present case, the Reynolds stress levels were affected by the longitudinal vortex and increased compared to the case without interaction, indicating that diffusion had increased. In the outflow region, the shear layer located in the formation area of the leading edge vortex is increased, leading to a "symmetricalisation" of the flow in the formation region.

\subsection{Phase portrait and phase averaging}

Taking into account that the motion at large scale varies cyclically with time, following the work presented by Ben Chiekh et al. (2004), we conducted an analysis of the two first coefficients $a_{1}$ and $a_{2}$ normalized by their standard deviations $\sigma_{a 1}$ and $\sigma_{a 2}$ respectively. The representation in the $\left(a_{1} / \sigma_{a 1}, a_{2} / \sigma_{a 2}\right)$ plane shows that the coefficients are not statistically independent and generally provide a series of Lissajou curves. In the experimental case, dispersion is observed due to the high Reynolds number value, noise measurements and high level of turbulence at small scales. The distribution is presented in Fig. 20(a). The two coefficients are distributed in a cloud of points in the form of a circle. We define a radius $r$ and a phase $\varphi$ by

$$
r=\sqrt{a_{1}^{2} / \sigma_{a_{1}}^{2}+a_{2}^{2} / \sigma_{a_{2}}^{2}}, \quad \tan (\phi)=\frac{a_{2} / \sigma_{a_{2}}}{a_{1} / \sigma_{a_{1}}} .
$$

Fig. 20(b) shows the distribution of each coefficient, $a_{1}$ and $a_{2}$, as a function of phase $\varphi$. Despite the dispersion, a quasisinusoidal evolution is observed as expected and the two coefficients are a quarter period apart.

As suggested by Ben Chiekh et al. (2004), Perrin et al. (2007) and Deri et al. (2013) determination of the phase can be used to represent instantaneous quantities such as vorticity during the shedding cycle. The vortex shedding was modulo $2 \pi$ and the period was segmented into 72 intervals. Between 37 and 48 instantaneous fields were considered each phase average. Figs. 21-24 show the phase averaged $\omega_{12}$ iso-contours without vortex interaction in the outflow area, using the phase defined with POD coefficients for $\varphi=\left(0^{\circ}, 90^{\circ}, 180^{\circ}, 270^{\circ}\right)$. The streamlines were superimposed.
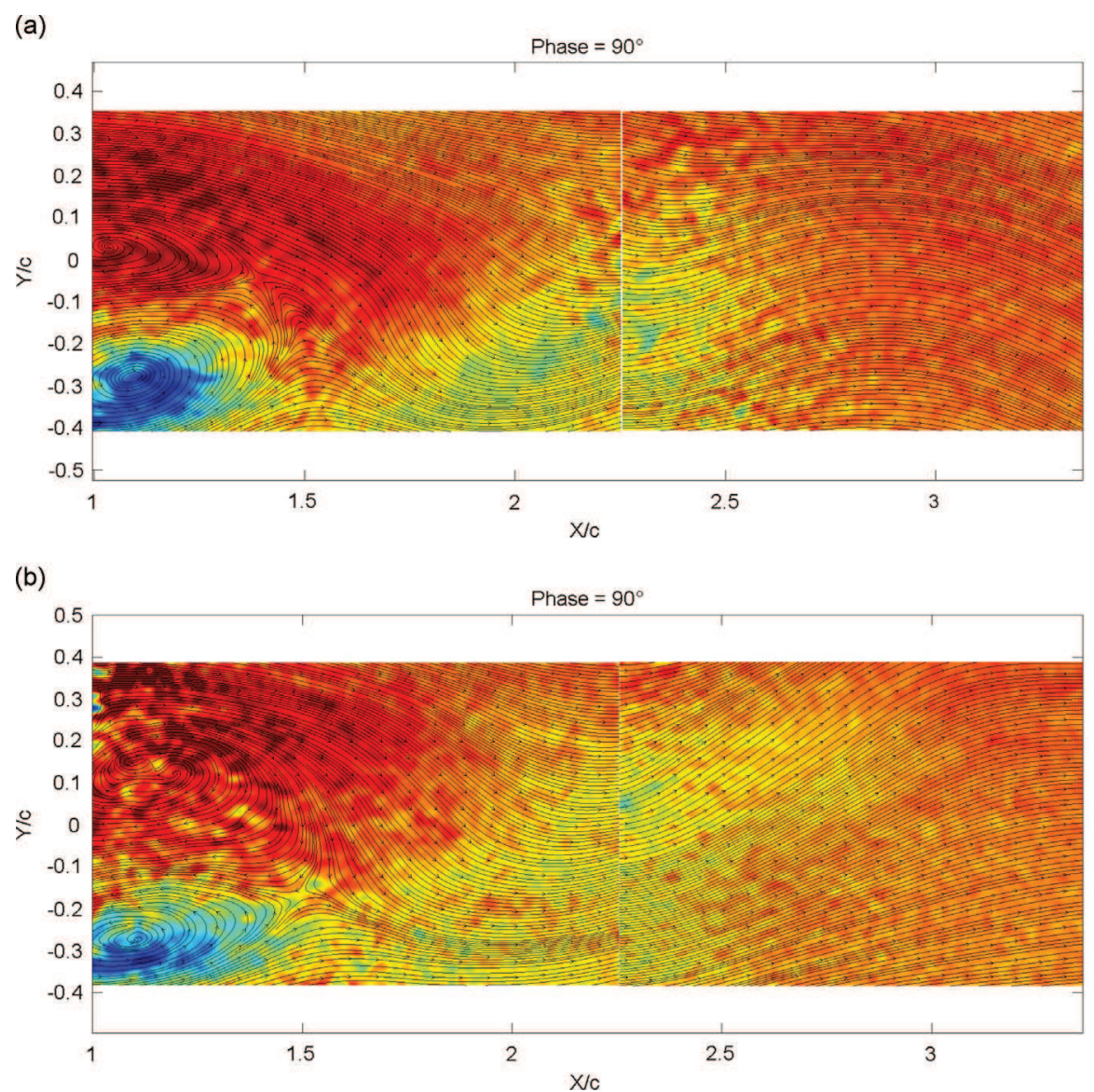

Fig. 22. $90^{\circ}$ Phase averaged vorticity obtained using the POD coefficients: (a) without upstream vortex and (b) with upstream vortex. 
(a)

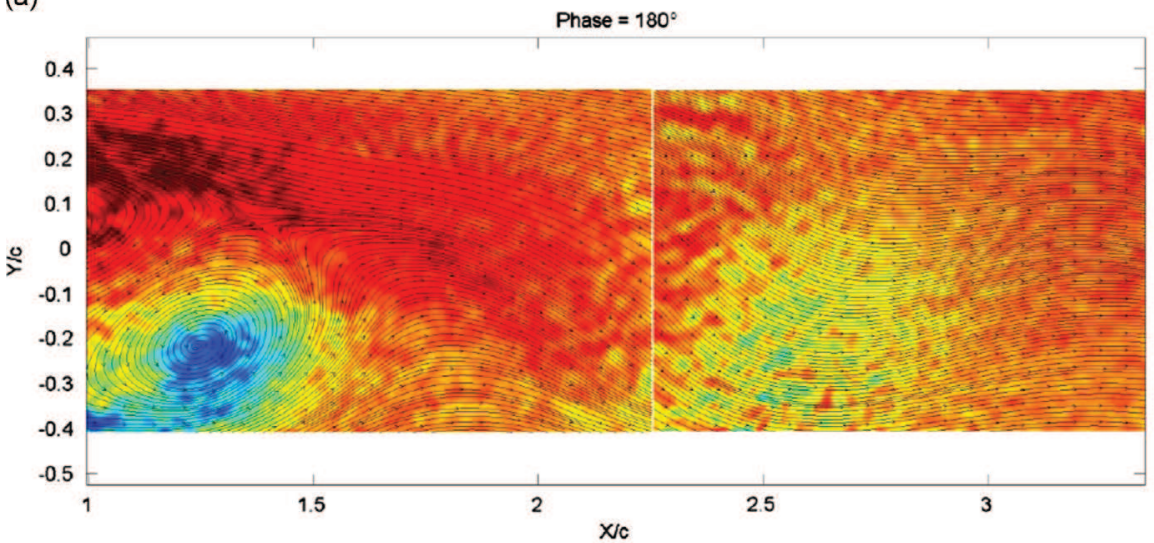

(b)

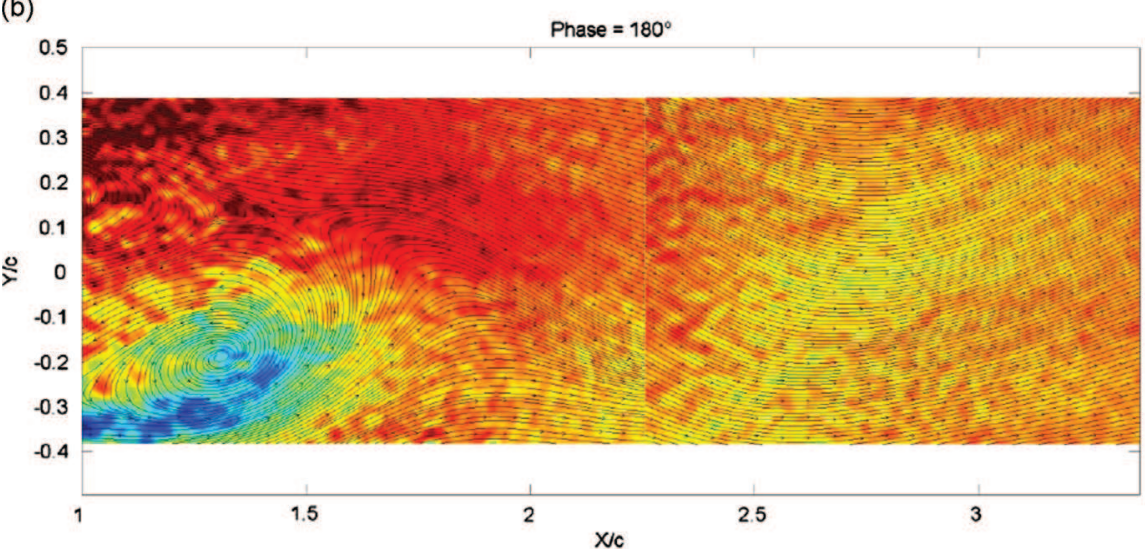

Fig. 23. $180^{\circ}$ Phase averaged vorticity obtained using the POD coefficients: (a) without upstream vortex and (b) with upstream vortex.

The topology is constituted by alternating positive and negative vorticity with an asymmetric aspect due to the alternating leading and trailing edge vortices. Without the longitudinal vortex, for $\varphi=0$ phase, the leading edge vortex is dominant in intensity over the trailing edge vortex. In the outflow area, in the same phase, the trailing edge vortex is more pronounced with an enlarged vorticity region with a decrease in intensity near the vortex core. Interactions are visible via a modification of the rotational activity which extends further into the wake of the NACA0015 foil leading to a symmetrization of the shedding. This behavior leads to an increase in the pressure fluctuations and hence a large modification of the lift and drag coefficients observed in the presence of the upstream longitudinal vortex.

\section{Summary and conclusions}

The present study analyzed the effects of the upstream single longitudinal vortex on wake dynamics of the NACA0015 foil at high incidence. The flow measurements were taken using TR-SPIV system in the near and far wake of a 2-D foil in the presence of the longitudinal vortex generated upstream by a 3-D NACA0020 foil at Reynolds number of $5 \times 10^{5}$. Particular attention was paid to the detachment at $25^{\circ}$ incidence and flow properties such as Reynolds stresses, vorticty, kinetic energy and turbulent production were obtained and discussed. Proper Orthogonal Decomposition (POD) analysis was applied in order to highlight the unsteady flow aspects using phase averaging while using the first POD coefficients to characterize the influence of the longitudinal vortex on the coherent process in the near wake of the rudder.

The comparative analyses of the results with and without upstream vortex indicate a particular effect on the vortex shedding at high incidences. The presence of the vortex inhibits the hysteresis loop and a plateau value is observed associated with an increase in lift associated with inhibition of the oscillations of the detachment point. In the presence of the upstream vortex, the interaction is characterized by an inhibition of the vortex shedding in the inflow area of the NACA0015 wake and a change in the saddle point location where the vortex induces a velocity component directed from the wall into the undisturbed flow (outflow area). Interaction in the outflow area is characterized by an expansion of the wake in the downstream direction, which is associated with a thickening effect in the vertical direction. Concerning the turbulent agitation, the turbulent production was inhibited in the inflow area and enhanced in the outflow area. A global decrease in the turbulent quantities was observed in the presence of the longitudinal vortex. 
(a)

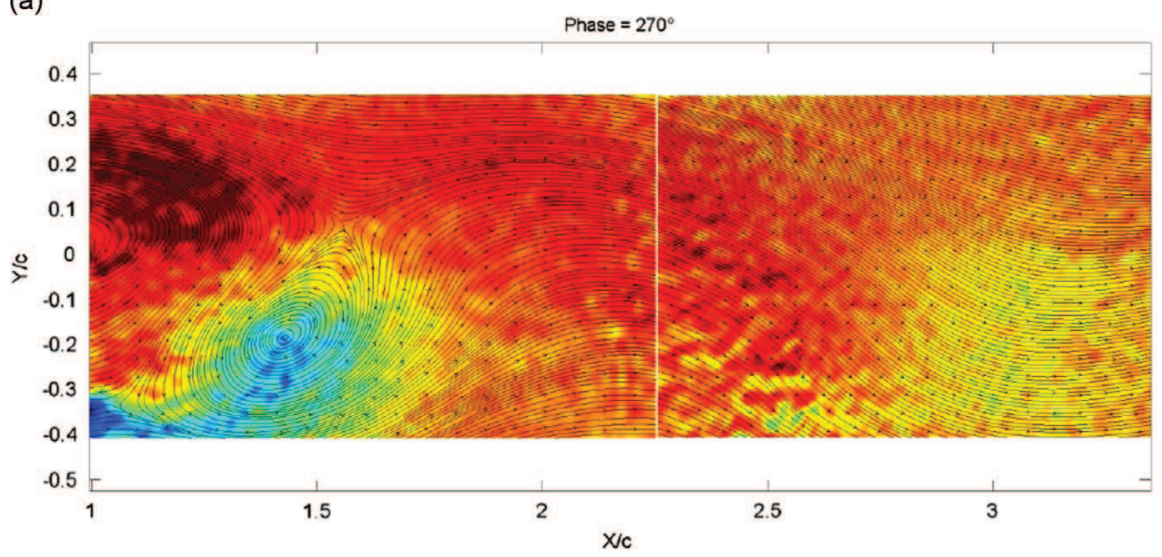

(b)

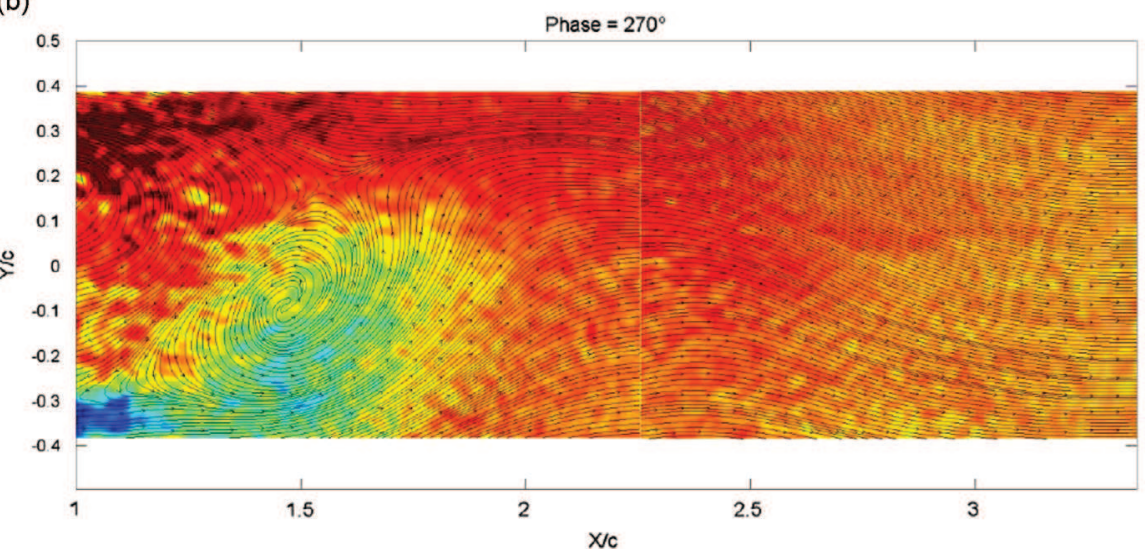

Fig. 24. $270^{\circ}$ Phase averaged vorticity obtained using the POD coefficients: (a) without upstream vortex and (b) with upstream vortex.

The eigenvectors of the first and second modes indicate that the Kármán-type structure is destructured in the "inflow" area corresponding to low distributions of the modal energy. In the "outflow" area (where the vortex induces a velocity directed upward of the foil) the spatial distribution of the first energetic modes is deviated due to the momentum exchange and the trailing vortex is not intense enough to exhibit realignment in the near wake. The comparative phase averaged vorticty provided an interaction of the upstream vortex on the wake area leading to a global symmetrization of the wake.

\section{References}

Angele, K.P., Muhammad-Klingmann, B., 2005. The effect of streamwise vortices on the turbulence structure of a separating boundary layer. European Journal of Mechanics, B/Fluids 24, 539-554.

Ben Chiekh, M., Michard, M., Grosjean, N., Bera, J.C., 2004. Reconstruction temporelle d'un champ aérodynamique instationnaire à partir de mesures PIV non résolues dans le temps. 9ième Congrès Francophone de Technique Laser, Brussels.

Berkooz, G., Holmes, P., Lumley, J.L., 1993. The proper orthogonal decomposition in the analysis of turbulent flows. Annual Review of Fluids Mechanics 25, 539-575.

Deri, E., Braza, M., Cid, E., Cazin, S., Michaelis, D., Degouet, C., 2013. Investigation of the three-dimensional turbulent near-wake structure past a flat plate by tomographic PIV at high Reynolds number. Journal of Fluids and Structures. http://dx.doi.org/10.1016/j.jfluidstructs.2012.11.005.

Djeridi, H., Braza, M., Perrin, R., Harran, G., Cid, E., Cazin, S., 2003. Near wake turbulence around a circular cylinder at high Reynolds number. Journal of Flow Turbulence and Combustion 71, 19-34.

Ducoin, A., Astolfi, J.A., Gobert, M.L., 2012. An experimental study of boundary-layer transition induced vibrations on a hydrofoil. Journal of Fluids and Structures 32, 37-51.

Felli, M., Camussi, R., Guj, G., 2009. Experimental analysis of the flow field around a propeller rudder configuration. Experiments in Fluids 46 (1), 147-164. Felli, M., Di Felice, F., Guj, G., Camussi, R., 2006. Analysis of the propeller wake evolution by pressure and velocity phase measurements. Experiments in Fluids 1, 1-11.

Felli, M., Falchi, M., Pereira, F., 2011. Investigation of the flow field around a propeller rudder configuration: on-surface pressure measurements and velocity pressure phase-locked correlations. In: Proceedings of the Second International Symposium on Marine Propulsors smp'11, Hamburg, Germany.

Hoffmann, J.A., 1991. Effects of freestream turbulence on the performance characteristics of an airfoil. AIAA Journal 29 (9), $1353-1354$.

Kinnas, S.A., Mohammed, F., Lee, H., 2006. Propeller tip vortex-rudder interaction. In: Proceedings of the 26th Symposium on Naval Hydrodynamics, Rome.

Mittal, S., Saxena, P., 2000. Prediction of hysteresis associated with the static stall of an airfoil. AIAA Journal 38 (5), $933-935$.

Noack, B., Afanasiev, K., Morzynsky, M., Tadmor, G., Thielle, F., 2003. A hierarchy of low-dimensionnal models for the transcient and post-transient cylinder wake. Journal of Fluids Mechanics 497, 335-363.

Norberg, C., 2003. Fluctuating lift on a circular cylinder: review and new measurements. Journal of Fluids and Structures 17 (1), 57-96. 
Perrin, R., Cid, E., Cazin, S., Sevrain, A., Braza, M., 2007. Phase-averaged measurements of the turbulence properties in the near wake of a circular cylinder at high Reynolds number by 2C-PIV and 3C-PIV. Experiments in Fluids 42 (1), 93-109.

Pichon, T. 1995. Contribution à l'étude de la cavitation de tourbillon marginal (Thèse de doctorat). Ecole Centrale de Nantes - Université de Nantes, IRENav. Prothin, S., 2011. Interaction Tourbillon/Couche limite. Editions Universitaires Européennes, ISBN: 978-613-1-56645-5.

Sarraf, C., Djeridi, H., Prothin, S., Billard, J.Y., 2010. Thickness effect of NACA foils on hydrodynamic global parameters, boundary layer states and stall establishment. Journal of Fluids and Structures 26 (4), 559-578.

Shen, Y.T., Jiang, C.W., Remmers, K.D., 1997. Effect of ship hull and propeller on rudder cavitation. Journal of Ship Research 41 (3) 172-180.

Simpson, R.L., 1989. Turbulent boundary-layer separation. Annual Review of Fluid Mechanics 21, 205-232.

Sirovich, L., 1987. Turbulence and the dynamics of coherent structures. Quarterly of Applied Mathematics 45, 561-590.

Tsakonas, S., Jacobs, W.R., Ali, M.R., 1970. Application of the unsteady lifting surface theory to the study of propeller rudder interaction. Journal of Ship Research 14 (3), 181-194.

Ünal, U.O., Atlar, M., 2010. An experimental investigation into the effect of vortex generators on the near-wake flow of a circular cylinder. Experiments in Fluids 48, 1059-4079.

Van Oudheusden, B.W., Scarano, F., Van Hinsberg, N.P., Watt, D.W., 2005. Phase-resolved characterization of vortex shedding in the near wake of a squaresection cylinder at incidence. Experiments in Fluids 39 (1), 86-98.

Zhu, Z.L., Dong, S.T., 1986. A theoretical method for predicting the hydrodynamic performances of propeller rudder interaction. In: Proceedings of the International Symposium on Propeller and Cavitation, Wuxi, China. 